Centre interuniversitaire de recherche en économie quantitative

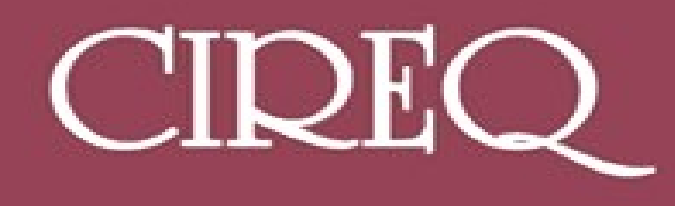

\title{
Cahier 17-2003
}

Methods to Estimate Dynamic Stochastic

General Equilibrium Models

Francisco J. RUGE-MURCIA 


\section{CIREQ}

Le Centre interuniversitaire de recherche en économie quantitative (CIREQ) regroupe des chercheurs dans les domaines de l'économétrie, la théorie économique, la macroéconomie et les marchés financiers, l'économie du travail et l'économie de l'environnement. Ils proviennent principalement des universités de Montréal, McGill et Concordia. Le CIREQ offre un milieu dynamique de recherche en économie quantitative grâce au grand nombre d'activités qu'il organise (séminaires, ateliers, colloques) et de collaborateurs qu'il reçoit chaque année.

The Center for Interuniversity Research in Quantitative Economics (CIREQ) regroups researchers in the fields of econometrics, economic theory, macroeconomics and financial markets, labor economics, and environmental economics. They come mainly from the Université de Montréal, McGill University and Concordia University. CIREQ offers a dynamic environment of research in quantitative economics thanks to the large number of activities that it organizes (seminars, workshops, conferences) and to the visitors it receives every year.

\section{Cahier 17-2003}

\section{Methods to Estimate Dynamic Stochastic General Equilibrium Models}

Francisco J. RUGE-MURCIA

téléphone : (514) 343-6557 télécopieur : (514) 343-5831 cireq@umontreal.ca http://www.cireq.umontreal.ca

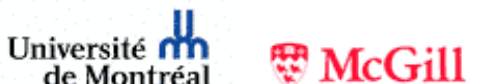
Concordia 
Ce cahier a également été publié par le Département de sciences économiques de l’Université de Montréal sous le numéro 2003-23.

This working paper was also published by the Département de sciences économiques of the Université de Montréal, under number 2003-23.

Dépôt légal, Bibliothèque nationale du Canada, 2003, ISSN 0821-4441

Dépôt légal, Bibliothèque nationale du Québec, 2003, ISBN 2-89382-475-7 


\title{
Methods to Estimate Dynamic Stochastic General Equilibrium Models*
}

\author{
Francisco J. Ruge-Murcia \\ Département de sciences économiques and C.I.R.E.Q., \\ Université de Montréal \\ First Draft: August 2002 \\ This Copy: June 2003
}

\begin{abstract}
This paper employs the one-sector Real Business Cycle model as a testing ground for four different procedures to estimate Dynamic Stochastic General Equilibrium (DSGE) models. The procedures are: 1) Maximum Likelihood, with and without measurement errors and incorporating Bayesian priors, 2) Generalized Method of Moments, 3) Simulated Method of Moments, and 4) Indirect Inference. Monte Carlo analysis indicates that all procedures deliver reasonably good estimates under the null hypothesis. However, there are substantial differences in statistical and computational efficiency in the small samples currently available to estimate DSGE models. GMM and SMM appear to be more robust to misspecification than the alternative procedures. The implications of the stochastic singularity of DSGE models for each estimation method are fully discussed.
\end{abstract}

JEL Classification: E13, C11, C13, C15, C32

Key Words: DSGE models, estimation methods, Monte Carlo analysis, stochastic singularity, Bayesian priors

\footnotetext{
${ }^{*}$ I have benefitted from conversations with Rui Castro, Wouter den Haan, Jesús Fernandez-Villaverde, Jim Hamilton, Frank Schorfheide, and Chris Sims. This project was completed while I was Visiting Professor at the University of California at San Diego. I wish to thank the Department of Economics at UCSD for its hospitality. Financial support from the Social Sciences and Humanities Research Council and the Fonds pour la Formation de Chercheurs et l'Aide à la Recherche is gratefully acknowledged. Correspondence: Départemente de sciences économiques, Université de Montréal, C.P. 6128, succursale Centre-ville, Montréal (Québec) H3C 3J7, Canada. E-mail: francisco.ruge-murcia@umontreal.ca
} 


\section{Résumé}

Cet article utilise un modèle de cycles réels à un secteur pour tester la validité et l'efficacité de quatre procédures d'estimation des modèles d'équilibre général dynamique stochastique (EGDS): 1) estimation par maximum de vraisemblance avec ou sans erreur de mesure et en introduisant un cadre bayésien à l'aide de distributions a priori; 2) par méthode des moments généralisée; 3) par méthode des moments simulés; et enfin, 4) par inférence indirecte. Des simulations de Monte-Carlo montrent que toutes ces méthodes produisent des estimateurs généralement bons sous l'hypothèse nulle. Cependant, leurs efficacités statistiques et computationnelles diffèrent de façon substantielle, en particulier lorsque l'on se place dans de petits échantillons comme ceux généralement disponibles pour estimer ce type de modèle. Dans ce cas, les méthodes des moments semblent être les plus robustes à une mauvaise spécification du modèle. Enfin, nous discutons, de manière exhaustive, de l'implication de la singularité stochastique des modèles EGDS pour chaque méthode d'estimation.

Mots clés: modèles EGDS, méthodes d'estimation, simulations de Monte-Carlo, singularité stochastique, cadre bayésien 


\section{Introduction}

Dynamic Stochastic General Equilibrium (DSGE) models have become a standard tool in various fields of Economics, most notably in Macroeconomics and International Economics. DSGE models are attractive because they specify explicitly the objectives and constraints faced by households and firms, and then determine the prices and allocations that result from their market interaction in an uncertain environment.

To date, calibration is by far the most common approach in the literature to examine the empirical properties of DSGE models. In calibration, the value of the structural parameters is fixed to those estimated in previous microeconometric studies and/or those computed using long-run averages of aggregate data. Then, the model is simulated using a synthetic series of shocks, and the unconditional moments of the simulated economic series are computed and compared with the ones of actual data. The model is usually evaluated in terms of the distance between these two set of moments. This comparison can be casual or based on measures of fit like the ones proposed, for example, by Gregory and Smith (1991), Watson (1993), and DeJong, Ingram, and Whiteman (1996). Impulse-response analysis and variance decomposition are also used to examine, respectively, the model's behavior following exogenous shocks and to assess the relative importance of these shocks in explaining the conditional and unconditional variances of the variables.

Although calibration is a very useful tool for understanding the dynamic properties of DSGE models, there are some advantages in their fully-fledged econometric estimation. First, parameter estimates are obtained by imposing on the data the restrictions of the model of interest. This addresses the concern that the assumptions of the DSGE model might be inconsistent with the assumptions employed by the micro studies that produced the parameter estimates used in calibration. Second, the estimation of the DSGE model allows one to obtain estimates of parameters that might be hard to estimate using disaggregated data alone. Third, parameter uncertainty can be incorporated explicitly in impulse-response analysis using, for example, bootstrap techniques to construct confidence intervals for the model's response to a shock. Finally, standard tools of model selection and evaluation can be readily applied. For example, one can test the residuals for serial correlation and neglected Autoregressive Conditional Heteroskedasticity, compare the Root Mean Square Error of the DSGE model with that of another DSGE model or a Vector Autoregression, perform tests of parameter stability or directly test some of the model's identification assumptions. All this is valuable information in the construction of more realistic economic models. ${ }^{1}$

\footnotetext{
${ }^{1}$ See Hansen and Heckman (1996), and Browning, Hansen, and Heckman (1999) for additional discussion. For a defense of the merits of calibration, see Kydland and Prescott (1996).
} 
The estimation procedures studied here are Maximum Likelihood (ML), Generalized Method of Moments (GMM), Simulated Method of Moments (SMM), and the Indirect Inference procedure proposed by Smith (1993). All these procedures are standard and their asymptotic properties are well know. The goals of this paper are to describe in a pedagogical manner their application to the estimation of DSGE models, to study their small-sample properties, to examine their robustness to misspecification, to compare their computational costs, and to discuss fully the implications of the stochastic singularity of DSGE models for each estimation procedure. The intention here is not to perform a "horse race" between different estimation strategies. Instead, the more constructive goal is to evaluate their relative strengths and weaknesses in the context of a simple, but economically interesting model.

An important feature of DSGE models that has implications for all estimation procedures is their stochastic singularity. DSGE models are usually singular because they generate predictions about a larger number of observable endogenous variables than exogenous shocks are used to feed the model. This means that there are linear combinations of the variables that hold without noise. These restrictions of the theoretical model arise from a particular form of misspecification: the model assumes a smaller number of shocks than are present in the real world.

Stochastic singularity limits the number of variables/moments that can be exploited for the estimation of the model, and imposes restrictions on both the order and the number of variables in the VAR representation of data generated by a DSGE model. In general, singularity affects more severely Maximum Likelihood than the Methods of Moments (that is, GMM and SMM). For example, the RBC model studied here cannot be estimated by Maximum Likelihood using more than one variable, unless measurement errors are added, but it can be estimated by the Methods of Moments using moments that involve more than one variable. Hence, the Methods of Moments might yield more precise parameter estimates than Maximum Likelihood, despite the fact that they are limited information procedures. The paper studies the effect of adding measurement errors to sidestep the singularity of the model and the use of Bayesian priors that incorporate information from microeconometric studies, long-run averages of aggregate data, and/or economic theory. Priors are incorporated here into the Maximum Likelihood framework using the mixed estimation strategy in Theil and Goldberger (1961) and are shown to yield sharper estimates than those obtained by the classical Maximum Likelihood estimator.

The paper is organized as follows. Section 2 describes the DSGE model that will be used as backdrop for the estimation procedures. Section 3 describes the estimation procedures and their application to DSGE models. Section 4 presents the Monte Carlo design and report its results. Section 5 concludes. 


\section{The Artificial Economy}

The discussion of the different estimation procedures is best made in the context of a specific economic model. This paper employs a version of the well-known one-sector Real Business Cycle model with indivisible labor [see Hansen (1985), and King, Plosser and Rebelo (1988)]. ${ }^{2}$ The representative agent maximizes expected lifetime utility defined by:

$$
U_{t}=E_{t} \sum_{i=t}^{\infty} \beta^{i-t}\left[\ln \left(c_{i}\right)+\psi \ell_{i}\right],
$$

where $\beta \in(0,1)$ is the subjective discount factor, $c_{t}$ is consumption, $\ell_{t}$ is leisure, and $\psi$ is the weight of leisure in the instantaneous utility function. There is no population growth. Without loss of generality, the population size and time endowment are normalized to one. Hence,

$$
n_{t}=1-\ell_{t}
$$

where $n_{t}$ is hours worked. The agent's income consists of wages and rents received from selling labor and renting capital to firms, and is allocated to consumption and investment:

$$
c_{t}+x_{t}=w_{t} n_{t}+r_{t} k_{t}
$$

where $x_{t}$ is investment, $w_{t}$ is the real wage, $r_{t}$ is the real rental rate of capital, and $k_{t}$ is the capital stock. The prices $w_{t}$ and $r_{t}$ are expressed in terms of units of the consumption good. Investment increases the stock of capital according to:

$$
k_{t+1}=(1-\delta) k_{t}+x_{t}
$$

where $\delta \in(0,1)$ is the depreciation rate. In addition to the transversality condition, the first-order necessary conditions associated with the optimal choice of $c_{t}, n_{t}$, and $k_{t+1}$ for this problem are:

$$
\begin{aligned}
1 / c_{t} & =\beta E_{t}\left[\left(1 / c_{t+1}\right)\left(1+r_{t}-\delta\right)\right] \\
\psi c_{t} & =w_{t}
\end{aligned}
$$

The single, perishable good in this economy is produced by perfectly competitive firms using a constant returns to scale technology. Since in this setup the number of firms

\footnotetext{
${ }^{2}$ In preliminary work, I performed a limited number of Monte-Carlo experiments using a more complicated DSGE model with money in the utility function, monopolistic competition, price rigidity, and adjustment costs to the capital stock. Conclusions are qualitatively similar to those reported here, but the complexity of the model obscures some of the points illustrated below.
} 
in equilibrium is indeterminate, it is convenient to focus on a representative firm. The representative firm rents labor and capital from the agent and combines them according to:

$$
y_{t}=z_{t}\left(k_{t}\right)^{\alpha}\left(n_{t}\right)^{1-\alpha}
$$

where $\alpha \in(0,1), y_{t}$ is output and $z_{t}$ is a technology shock. The technology shock follows the exogenous stochastic process:

$$
\ln z_{t+1}=\rho \ln z_{t}+\epsilon_{t}
$$

where $\rho \in(-1,1)$ and $\epsilon_{t}$ is an innovation assumed to be independently, identically, and Normally distributed with zero mean and variance $\sigma^{2}$. In every period, the firm chooses input levels to maximize profits and equates the marginal product of labor (capital) to the real wage (rental rate). Due to the assumptions of perfect competition and constant returns to scale, firms make zero profits in equilibrium.

The competitive equilibrium for this economy is the sequence of prices $\left\{w_{t}, r_{t}\right\}_{t=0}^{\infty}$ and allocations $\left\{c_{t}, n_{t}, x_{t}, k_{t+1}, y_{t}\right\}_{t=0}^{\infty}$ such that firms maximize profits, agents maximize utility, and all markets clear. In particular, goods market clearing requires that aggregate output be equal to aggregate demand:

$$
y_{t}=c_{t}+x_{t}
$$

The usual strategy to solve DSGE models involves the linearization of first-order conditions and constraints by means of a first-order Taylor series expansion around the model's steady state. The linearized equations for this model are presented in the Appendix. These equations form a dynamic system that determines the path of the six variables in the model, namely consumption, capital, output, investment, hours worked, and the technology shock. ${ }^{3}$ Using the circumflex to denote percentage deviation from steady state and after some manipulations, it is possible to write:

$$
\left[\begin{array}{c}
\hat{k}_{t+1} \\
E_{t} \hat{c}_{t+1}
\end{array}\right]=\mathbf{A}\left[\begin{array}{c}
\hat{k}_{t} \\
\hat{c}_{t}
\end{array}\right]+\mathbf{B} \hat{z}_{t}
$$

where

$$
\begin{aligned}
& \mathbf{A}=\left[\begin{array}{cc}
a_{11} & a_{12} \\
0 & a_{22}
\end{array}\right]=\left[\begin{array}{cc}
1+\delta \gamma /(1-\gamma) & -\delta(1+\alpha \gamma-\alpha) /(\alpha-\alpha \gamma) \\
0 & \alpha /(\varsigma+\alpha-\alpha \varsigma)
\end{array}\right] \\
& \mathbf{B}=\left[\begin{array}{l}
b_{1} \\
b_{2}
\end{array}\right]=\left[\begin{array}{c}
\delta /(\alpha-\alpha \gamma) \\
\varsigma \rho /(\varsigma+\alpha-\alpha \varsigma)
\end{array}\right],
\end{aligned}
$$

\footnotetext{
${ }^{3}$ For convenience and without loss of generality, I have substituted out the wage and rental rate by equating them to the marginal products of labor and capital, respectively.
} 
$\varsigma=\alpha \beta(k / n)^{\alpha-1}, k / n=((1 / \beta+\delta-1) / \alpha)^{1 /(\alpha-1)}$ is the steady-state capital-labor ratio, $\gamma=1-\delta(k / n)^{1-\alpha}$ is the steady-state consumption-output ratio, and variables without time subscript denote steady state values. The rational expectations solution of this system can be found using, for example, the approaches proposed by Blanchard and Kahn (1980) and Sims (1997) to obtain:

$$
\begin{aligned}
\hat{k}_{t+1} & =a_{11} \hat{k}_{t}+a_{12} \hat{c}_{t}+b_{1} \hat{z}_{t}, \\
\hat{c}_{t} & =\phi_{c k} \hat{k}_{t}+\phi_{c z} \hat{z}_{t}
\end{aligned}
$$

where $\phi_{c k}$ and $\phi_{c z}$ are combinations of the eigenvectors and eigenvalues of the matrix $\mathbf{A}$ and, consequently, depend nonlinearly on the structural parameters.

In what follows, it is convenient to define the $2 \times 1$ vector $\boldsymbol{\xi}_{t}=\left(\hat{k}_{t}, \hat{z}_{t}\right)^{\prime}$ that collects the state variables of the system, and the $3 \times 1$ vector $\mathbf{s}_{t}=\left(\hat{n}_{t}, \hat{y}_{t}, \hat{c}_{t}\right)^{\prime}$ that contains the observable variables that the researcher will use later in the estimation of the model. Using the linearized equations of the model, it is possible to write the components of $\mathbf{s}_{t}$ as functions of the capital stock and technology shock alone:

$$
\mathbf{s}_{t}=\left[\begin{array}{c}
\hat{y}_{t} \\
\hat{n}_{t} \\
\hat{c}_{t}
\end{array}\right]=\boldsymbol{\Phi} \boldsymbol{\xi}_{t}=\left[\begin{array}{cc}
\phi_{y k} & \phi_{y z} \\
\phi_{n k} & \phi_{n z} \\
\phi_{c k} & \phi_{c z}
\end{array}\right]\left[\begin{array}{c}
\hat{k}_{t} \\
\hat{z}_{t}
\end{array}\right]
$$

The last row of $\mathbf{s}_{t}$ (i.e., the expression for consumption) reproduces exactly equation (2). Finally, notice that the elements of the $3 \times 2$ matrix $\Phi$ are nonlinear functions of the structural parameters of the model.

This model takes as input the predetermined level of capital and one exogenous shock, and generates predictions about (at least) three observable endogenous variables, namely output, consumption and hours worked. Since the number of shocks is less than the number of endogenous variables, there are linear combinations of these variables that are predicted to hold without noise. Hence, the model is stochastically singular. For example, one can eliminate both $\hat{k}_{t}$ and $\hat{z}_{t}$ from (3) to obtain:

$$
\left(\phi_{y k} \phi_{c z}-\phi_{y z} \phi_{c k}\right) \hat{n}_{t}+\left(\phi_{n z} \phi_{c k}-\phi_{n k} \phi_{c z}\right) \hat{y}_{t}-\left(\phi_{n z} \phi_{y k}-\phi_{y z} \phi_{n k}\right) \hat{c}_{t}=0
$$

Similarly, using the equations for $\hat{y}_{t}$ and $\hat{c}_{t}$ in $(3)$ and the linearized law of motion for capital, it is possible to write

$$
\begin{aligned}
& {\left[\phi_{y z}+\delta \gamma\left(\phi_{y z} \phi_{c k}-\phi_{y k} \phi_{c z}\right) /(1-\gamma)\right] \hat{c}_{t}-(1-\delta) \phi_{y z} \hat{c}_{t-1}} \\
& -\left[\phi_{c z}+\delta\left(\phi_{y z} \phi_{c k}-\phi_{y k} \phi_{c z}\right) /(1-\gamma)\right] \hat{y}_{t}+(1-\delta) \phi_{c z} \hat{y}_{t-1}=0 .
\end{aligned}
$$

Combining (4) and (5), it is easy to show that the systems $\left(\hat{y}_{t}, \hat{n}_{t}, \hat{y}_{t-1}, \hat{n}_{t-1}\right)$ and $\left(\hat{n}_{t}, \hat{c}_{t}\right.$, $\left.\hat{n}_{t-1}, \hat{c}_{t-1}\right)$ are also singular. That is, for any sample size, the sample variance-covariance 
matrix of these systems are singular. We will see below that stochastic singularity has different and nontrivial implications for the application of each estimation method to DSGE models.

\section{Estimation Methods}

\subsection{Maximum Likelihood}

The Maximum Likelihood (ML) estimation of DSGE models requires the construction and evaluation of the likelihood function of the data given the structural parameters. This paper considers the more complicated case where the model involves unobserved state variables and filtering techniques are required to evaluate the likelihood function. In particular, I follow Ireland (1999) in treating the aggregate capital stock as unobservable. ${ }^{4}$ The case where the capital stock is treated as observable is a straightforward simplification of the setup considered here [see, for example, Christiano (1988), Altug (1989), and Bencivenga (1992)]. ${ }^{5}$

With unobservable state variables, one can exploit the recursive nature of the model and its fully-specified laws of motion to apply filters like the one proposed by Kalman (1960). ${ }^{6}$ The Kalman filter allows the construction of inferences about the unobserved state vector and permits the evaluation of the joint likelihood function of observable endogenous variables. In turn, the maximization of this likelihood function yields consistent and asymptotically normal estimates of the parameters of the model. This approach has been employed by, among others, McGrattan (1994), Hall (1996), McGrattan, Rogerson, and Wright (1997), Ireland (1999, 2001), Kim (2000), Bouakez, Cardia, and Ruge-Murcia (2001), and Dib and Phaneuf (2001).

The state-space representation of the DSGE model above consists of the following state and observation equations. The state equation is constructed by substituting (2) into (1) to obtain the law of motion of $\hat{k}_{t+1}$ in terms of $\hat{k}_{t}$ and $\hat{z}_{t}$ only, and by using the linearized process of the technology shock (see the last equation in the Appendix). Then, it is possible to write:

$$
\boldsymbol{\xi}_{t+1}=\mathbf{F} \boldsymbol{\xi}_{t}+\mathbf{v}_{t+1}
$$

\footnotetext{
${ }^{4}$ This assumption is made throughout the paper, so that differences in the Monte Carlo results across estimation methods are not due to the way in which the capital stock is treated.

${ }^{5}$ These authors employ variants of the ML procedure proposed by Hansen and Sargent (1980) to estimate general equilibrium models. Estimates of the U.S. capital stock are constructed using data on past investment and an educated guess about the rate of depreciation.

${ }^{6}$ As an alternative, Fernández-Villaverde and Rubio (2002) suggest the use of a nonlinear Sequential Monte Carlo filter to evaluate the log likelihood function of nonlinearized DSGE models.
} 
where

$$
\mathbf{F}=\left[\begin{array}{cc}
a_{11}+a_{12} \phi_{c k} & a_{12} \phi_{c z}+b_{1} \\
0 & \rho
\end{array}\right],
$$

is a $2 \times 2$ matrix and $\mathbf{v}_{t}=\left(0, \boldsymbol{\epsilon}_{t}\right)^{\prime}$ is a $2 \times 1$ vector. The observation equation consists of the process of one of the observable endogenous variables in (3):

$$
\mathbf{x}_{t}=\mathbf{h} \mathbf{s}_{t}=\mathbf{h} \Phi \boldsymbol{\xi}_{t}=\mathbf{H} \boldsymbol{\xi}_{t}
$$

where $\mathbf{h}$ is a $1 \times 3$ selection vector. For example, in the case where the model is estimated using output data alone, $\mathbf{h}=(1,0,0)$ and $\mathbf{x}_{t}=\hat{y}_{t}$. The reason why $\mathbf{x}_{t}$ contains only one variable will become clearer below.

Let us collect the structural parameters of the model in the $q \times 1$ vector $\boldsymbol{\theta}$. Denote by $\aleph_{t-1}=\left(\mathbf{x}_{t-1}, \mathbf{x}_{t-2}, \ldots, \mathbf{x}_{1}\right)$ the set of past observations of $\mathbf{x}_{t}$, by $\tilde{\boldsymbol{\xi}}_{t \mid t-1}$ the time $t-1$ forecast of $\boldsymbol{\xi}_{t}$ constructed on the basis of $\aleph_{t-1}$, and by $\mathbf{P}_{t \mid t-1}$ the Mean Square Error (MSE) of this forecast. Then, under the assumption that the technology innovation, $\epsilon_{t}$, is normally distributed, the density of $\mathbf{x}_{t}$ conditional on $\aleph_{t-1}$ is

$$
f\left(\mathbf{x}_{t} \mid \aleph_{t-1} ; \boldsymbol{\theta}\right)=N\left(\mathbf{H} \tilde{\boldsymbol{\xi}}_{t \mid t-1}, \mathbf{H P}_{t \mid t-1} \mathbf{H}^{\prime}\right)
$$

The Maximum Likelihood estimator of $\boldsymbol{\theta}$ is

$$
\tilde{\boldsymbol{\theta}}_{m l}=\max _{\{\boldsymbol{\theta}\}} L(\boldsymbol{\theta}),
$$

where $L(\boldsymbol{\theta})$ denotes the log likelihood function:

$$
\begin{aligned}
L(\boldsymbol{\theta})= & -(T / 2) \ln (2 \pi)-(1 / 2) \ln \left|\mathbf{H} \mathbf{P}_{t \mid t-1} \mathbf{H}^{\prime}\right| \\
& -(1 / 2) \sum_{i=1}^{T}\left(\mathbf{x}_{t}-\mathbf{H} \tilde{\boldsymbol{\xi}}_{t \mid t-1}\right)^{\prime}\left(\mathbf{H} \mathbf{P}_{t \mid t-1} \mathbf{H}^{\prime}\right)^{-1}\left(\mathbf{x}_{t}-\mathbf{H} \tilde{\boldsymbol{\xi}}_{t \mid t-1}\right),
\end{aligned}
$$

and $T$ is the sample size.

Since the process of $\boldsymbol{\xi}_{t}$ is stationary by construction, the Kalman filter recursion can be started with the unconditional moments $\tilde{\boldsymbol{\xi}}_{1 \mid 0}=E\left(\boldsymbol{\xi}_{t}\right)=(0,0)^{\prime}$ and $\mathbf{P}_{1 \mid 0}=E\left(\boldsymbol{\xi}_{t} \boldsymbol{\xi}_{t}^{\prime}\right)$. The subsequent forecasting and updating of $\boldsymbol{\xi}_{t}$ and the computation of the MSE of $\tilde{\boldsymbol{\xi}}_{t \mid t-1}$ are obtained using the Kalman algorithm described, for example, in Hamilton (1994, ch. 13). Under standard regularity conditions [see Judge et al. (1985, p. 178)], the ML estimator is consistent and asymptotically normal:

$$
\sqrt{T}\left(\tilde{\boldsymbol{\theta}}_{m l}-\boldsymbol{\theta}\right) \rightarrow N\left(\mathbf{0},(\Im / T)^{-1}\right),
$$

where $\Im=-E\left(\partial^{2} L(\boldsymbol{\theta}) / \partial \boldsymbol{\theta} \partial \boldsymbol{\theta}^{\prime}\right)$ is the information matrix. In the Monte Carlo, $\Im$ is estimated using the numerically computed Hessian of the log likelihood function at the optimum. 
Since there is only one random shock in this model, any attempt to estimate it by Maximum Likelihood using data on more than one variable yields a singular $\mathbf{H P}_{t \mid t-1} \mathbf{H}^{\prime}$ matrix. To see why, write the innovation in $\mathbf{x}_{t}$ :

$$
\mathbf{x}_{t}-E\left(\mathbf{x}_{t} \mid \aleph_{t-1}\right)=\mathbf{H}\left(\boldsymbol{\xi}_{t}-\tilde{\boldsymbol{\xi}}_{t \mid t-1}\right),
$$

and note that when $\mathbf{x}_{t}$ contains more than one variable, the innovations to the variables in $\mathbf{x}_{t}$ (say, consumption and output) are perfectly correlated and proportional to the technology shock. Thus, the matrix $\operatorname{Var}\left(\mathbf{x}_{t}-E\left(\mathbf{x}_{t} \mid \aleph_{t-1}\right)\right)=\mathbf{H P}_{t \mid t-1} \mathbf{H}^{\prime}$ is singular. In general, stochastically singular DSGE models cannot be estimated by Maximum Likelihood using more observable variables than structural shocks are specified in the model. For example, the RBC model studied here cannot be estimated by Maximum Likelihood using more than one variable. However, we will see below that it is possible to use data on up to two variables for the Methods of Moments and Indirect Inference. In this sense, stochastic singularity affects Maximum Likelihood more severely than alternative estimation methods.

There are at least two strategies to deal with singular DSGE models in the Maximum Likelihood framework. First, one can estimate the model using at most as many observable variables as structural shocks. This strategy is followed by Kim (2000), Ireland (2001), Bouakez, Cardia, and Ruge-Murcia (2001), and Dib and Phaneuf (2001). Second, one can add error terms to the observation equation of the state-space representation as in McGrattan (1994), Hall (1996), McGrattan, Rogerson, and Wright (1997) and Ireland (1999). ${ }^{7}$

\subsubsection{Adding Measurement Errors}

Adding extra error terms to the observation equation of the state-space representation of the DSGE model yields:

$$
\mathbf{x}_{t}=\mathbf{h} \mathbf{s}_{t}+\mathbf{u}_{t}=\mathbf{h} \Phi \boldsymbol{\xi}_{t}+\mathbf{u}_{t}=\mathbf{H} \boldsymbol{\xi}_{t}+\mathbf{u}_{t},
$$

where $\mathbf{x}_{t}$ is now a $d \times 1$ vector, $d$ is the number of observable variables used to estimate the model, $\mathbf{h}$ is a $d \times 3$ selection matrix, and $\mathbf{u}_{t}$ is a $d \times 1$ vector of shocks assumed independently, identically, and Normally distributed with zero mean and variance-covariance matrix

\footnotetext{
${ }^{7}$ Another alternative is to extend the model to permit additional structural shocks. This strategy is attractive because it increases the realism of the model and allows the use of more observable variables in the estimation of the model. Early advocates of this strategy include Leeper and Sims (1994) and Ingram, Kocherlakota, and Savin (1994). In general, adding structural shocks postpones, but does not necessarily solve, the stochastic singularity of DGSE models. For example, incorporating a government expenditure shock into the RBC model would add a second structural disturbance, but also would add another observable variable (i.e., government expenditure). However, adding structural shocks can solve the singularity of a model if a sufficient number of these shocks are latent. See Bergin (2003) for an application of this idea. Another issue is that while adding measurement errors preserves the original economic model, adding structural errors does not. The reason is that decision rules in the extended model would depend on a larger set of state variables than in the original model.
} 
$E\left(\mathbf{u}_{t} \mathbf{u}_{t}^{\prime}\right)=\mathbf{R}$. Based on Sargent (1989), it is customary to interpret $\mathbf{u}_{t}$ as measurement error. It is further assumed that $E\left(\mathbf{v}_{i} \mathbf{u}_{j}^{\prime}\right)=\mathbf{0}$ for all $i \geq j$, meaning that measurement error contains no information about current or future structural shocks. The extension to serially correlated errors is straightforward and is discussed in Hansen and Sargent (1998, ch. 8).

As before, the Maximum Likelihood estimator of $\boldsymbol{\theta}$ is

$$
\tilde{\boldsymbol{\theta}}_{m l}=\max _{\{\boldsymbol{\theta}\}} L(\boldsymbol{\theta}),
$$

but now the log likelihood function is:

$$
\begin{aligned}
L(\boldsymbol{\theta})= & -(T / 2) \ln (2 \pi)-(1 / 2) \ln \left|\mathbf{H P}_{t \mid t-1}^{\prime} \mathbf{H}^{\prime}+\mathbf{R}\right| \\
& -(1 / 2) \sum_{i=1}^{T}\left(\mathbf{x}_{t}-\mathbf{H} \boldsymbol{\xi}_{t}\right)^{\prime}\left(\mathbf{H P}_{t \mid t-1}^{\prime} \mathbf{H}^{\prime}+\mathbf{R}\right)^{-1}\left(\mathbf{x}_{t}-\mathbf{H} \boldsymbol{\xi}_{t}\right) .
\end{aligned}
$$

Notice that since (by assumption) $\mathbf{R}$ is full rank, then $\mathbf{H P}_{t \mid t-1}^{\prime} \mathbf{H}^{\prime}+\mathbf{R}$ will no longer be singular when the number of observable variables included in $\mathbf{x}_{t}$ is larger than the number of structural shocks.

One drawback of this approach is that measurement error lacks a truly structural interpretation and it essentially represents specification error. One can think of the singularity of DSGE models as arising from a particular form of misspecification: in the real world there are many more types of shocks than the ones assumed by the model. This is the main specification error that is captured by the term $\mathbf{u}_{t}$. When $\mathbf{u}_{t}$ is modeled as serially and/or contemporaneously correlated, other forms of misspecification can be captured by this term as well. Ireland (1999) interprets measurement errors as capturing movements and comovements in the data that the model cannot explain.

On the other hand, it can be argued that adding extra error terms is just a simple strategy to deal with misspecification. Relationships like (4) and (5) might hold approximately in the data, even if not without noise as predicted by the DSGE model. Thus, adding measurement error provides a less stringent platform to assess the theory.

\subsubsection{Incorporating Priors}

Economic theory, previous microeconometric studies, and long-run averages of aggregate data can be informative about the parameter values in structural macroeconomic models. This prior knowledge about $\boldsymbol{\theta}$ can be represented in a prior density and combined with aggregate time series data to obtain a posterior density of $\boldsymbol{\theta}$. The posterior density summarizes our knowledge about $\boldsymbol{\theta}$ after observing the data and is the basis of probabilistic statements regarding the structural parameters. There is a sense in which calibration can be interpreted as a Bayesian procedure where the prior density of $\boldsymbol{\theta}$ is degenerate and concentrated on a 
single numerical value. With such a strong prior, observations of the data series contribute nothing to our knowledge of the parameter values and the posterior density coincides with the prior one.

A simple way to incorporate priors into the Maximum Likelihood framework is based on the mixed estimation strategy in Theil and Goldberger (1961). This approach was originally developed for the linear regression model and leads to a Generalized Least Squares (GLS) estimator that incorporates optimally prior information regarding the parameter values. It is easy to show that the mean and variance of this GLS estimator corresponds exactly to mean and variance of the Bayesian posterior distribution [see Hamilton (1994, p. 359)]. Stone (1954) gives a Maximum Likelihood interpretation to the same estimator. DeJong, Ingram, and Whiteman (2000) and Chang, Gomes, and Schorfheide (2002) incorporate priors in the estimation of DSGE models and use, respectively, importance sampling and the MetropolisHastings algorithm to compute numerically the moments of the posterior distribution.

For the mixed estimation strategy, write the prior distribution of the parameters as

$$
\boldsymbol{\mu}=\mathbf{K} \boldsymbol{\theta}+\mathbf{e}
$$

where $\boldsymbol{\mu}$ is $q \times 1$ vector, $\mathbf{K}$ is a known $q \times q$ nonsingular matrix, and $\mathbf{e}$ is $q \times 1$ vector of random errors assumed Normally distributed with zero mean, variance-covariance matrix $\boldsymbol{\Sigma}$, and independent of $\mathbf{v}_{t}$ and $\mathbf{u}_{t}$. The matrix $\boldsymbol{\Sigma}$ is assumed known and represents the researcher's uncertainty about the prior information. This specification of the prior distribution is general in that it allows the characterization of the priors in terms of linear combinations of the parameters, and permits correlations across priors in the form of nonzero elements in the off-diagonal of $\boldsymbol{\Sigma}$. In the special case where $\mathbf{K}$ is diagonal, the prior would take the familiar form $f(\boldsymbol{\theta})=N(\boldsymbol{\mu}, \boldsymbol{\Sigma})$.

The mixed estimation strategy interprets the prior information as a set of $q$ additional observations of $\boldsymbol{\mu}$ and combines them with the sample of $T$ observations of the data $\aleph_{T}=\left(\mathbf{x}_{T}\right.$, $\left.\mathbf{x}_{T-1}, \mathbf{x}_{T-2}, \ldots, \mathbf{x}_{1}\right)$ to obtain an estimate of $\boldsymbol{\theta}$ as in:

$$
\tilde{\boldsymbol{\theta}}_{q b}=\max _{\{\boldsymbol{\theta}\}} L(\boldsymbol{\theta})+L(\boldsymbol{\mu}(\boldsymbol{\theta})) .
$$

where $L(\boldsymbol{\mu}(\boldsymbol{\theta}))$ is the $\log$ of the density of $\boldsymbol{\mu}$ in (8) and $L(\boldsymbol{\theta})$ was defined above. For the Monte Carlo, I will treat this quasi-Bayesian estimate of $\boldsymbol{\theta}$ as asymptotically normally distributed with variance-covariance matrix $(\Im)^{-1}$ and estimate the information matrix using the numerically computed Hessian at the optimum.

Notice that the estimator defined in (9) corresponds to the mode of the log of the posterior distribution $f\left(\boldsymbol{\theta} \mid \aleph_{T}\right)$. However, under the assumption of Gaussianity, the mode corresponds 
exactly to the mean and, consequently, the point estimate of $\boldsymbol{\theta}$ obtained here is the same as the one obtained using the approach in DeJong, Ingram, and Whiteman (2000). In the special case where the prior is diffuse or improper, the estimator $\tilde{\boldsymbol{\theta}}_{q b}$ converges to the classical ML estimator. When the prior is proper, the quasi-Bayesian estimate of $\boldsymbol{\theta}$ can be interpreted as the one obtained by the maximization of a penalized log likelihood function. The penalty $L(\boldsymbol{\mu}(\boldsymbol{\theta}))$ depends on the strength of the researcher's prior about $\boldsymbol{\theta}$ and has the effect of "pulling" the estimator towards the mean of the prior density.

\subsection{Simulated Method of Moments}

In calibration, the researcher computes the unconditional moments of synthetic series simulated using given parameter values and then compares them with the unconditional moments of the data. The Simulated Method of Moments (SMM) estimator pursues this idea further by updating the parameter values in a manner that reduces the distance between these two sets moments. SMM estimators have been proposed by McFadden (1989) and Pakes and Pollard (1989) to estimate discrete-choice problems and by Lee and Ingram (1991) and Duffie and Singleton (1993) to estimate time-series models.

Let us define $\mathbf{m}_{t}$ to be a $p \times 1$ vector of empirical observations on variables whose moments are of interest. Elements of $\mathbf{m}_{t}$ could include, for example, $\hat{y}_{t} \hat{c}_{t}, \hat{y}_{t} \hat{y}_{t-1}$, etc. Define $\mathbf{m}_{i}(\boldsymbol{\theta})$ to be the synthetic counterpart of $\mathbf{m}_{t}$ whose elements are computed on the basis of artificial data generated by the DSGE model using parameter values $\boldsymbol{\theta}$. The sample size is denoted by $T$ and the number of observations in the artificial time series is $\tau T$. The SMM estimator, $\tilde{\boldsymbol{\theta}}_{\text {smm }}$, is the value of $\boldsymbol{\theta}$ that solves

$$
\min _{\{\boldsymbol{\theta}\}} \mathbf{G}(\boldsymbol{\theta})^{\prime} \mathbf{W G}(\boldsymbol{\theta}),
$$

where

$$
\mathbf{G}(\boldsymbol{\theta})=(1 / T) \sum_{t=1}^{T} \mathbf{m}_{t}-(1 / \tau T) \sum_{i=1}^{\tau T} \mathbf{m}_{i}(\boldsymbol{\theta}),
$$

is a $p \times 1$ vector, and $\mathbf{W}$ is the optimal weighting matrix

$$
\mathbf{W}=\lim _{T \rightarrow \infty} \operatorname{Var}\left((1 / \sqrt{T}) \sum_{t=1}^{T} \mathbf{m}_{t}\right)^{-1} .
$$

Notice that, as usual, by using the optimal weighting matrix, a larger weight is given to the moments, or the linear combinations of moments, that are most informative.

Under the regularity conditions in Duffie and Singleton (1993),

$$
\sqrt{T}\left(\tilde{\boldsymbol{\theta}}_{s m m}-\boldsymbol{\theta}\right) \rightarrow N\left(\mathbf{0},(1+1 / \tau)\left(\mathbf{D}^{\prime} \mathbf{W}^{-1} \mathbf{D}\right)^{-1}\right),
$$


where $\mathbf{D}=E\left(\partial \mathbf{m}_{i}(\boldsymbol{\theta}) / \partial \boldsymbol{\theta}\right)$ is a $q \times p$ matrix assumed to be finite and of full rank. In the Monte Carlo, the derivatives $\partial \mathbf{m}_{i}(\boldsymbol{\theta}) / \partial \boldsymbol{\theta}$ are computed numerically and the expectation approximated by the average over the simulated $\tau T$ data points. $\mathbf{W}$ is computed using the Newey-West estimator with a Barlett kernel. A general specification test of the DSGE model can be constructed using the chi-square test proposed by Hansen (1982). As it is well known, this test can be applied provided that the model be overidentified, meaning $p>q$. In the case of SMM:

$$
T(1+1 / \tau)\left[\mathbf{G}\left(\tilde{\boldsymbol{\theta}}_{s m m}\right)^{\prime} \mathbf{W G}\left(\tilde{\boldsymbol{\theta}}_{s m m}\right)\right] \rightarrow \chi^{2}(p-q),
$$

where $\mathbf{G}\left(\tilde{\boldsymbol{\theta}}_{s m m}\right)^{\prime} \mathbf{W} \mathbf{G}\left(\tilde{\boldsymbol{\theta}}_{\text {smm }}\right)$ is the value of the objective function at the optimum.

The stochastic singularity of DSGE models also affects their estimation by SMM. In particular, singularity restricts the variables that can be included in the vector $\mathbf{m}$. For example, multiplying (4) by $\hat{y}_{t}$ shows that $\hat{n}_{t} \hat{y}_{t}, \hat{y}_{t}^{2}$, and $\hat{c}_{t} \hat{y}_{t}$ are not linearly independent. Thus, if one were to include these three variables as elements of $\mathbf{m}$, the Jacobian matrix $\mathbf{D}$ would not be of full rank and Assumption 6 in Duffie and Singleton (1993, p. 944) would not be satisfied. Similarly, multiplying (5) by $\hat{y}_{t}$ shows that $\hat{y}_{t}^{2}, \hat{c}_{t} \hat{y}_{t}, \hat{y}_{t-1} \hat{y}_{t}$, and $\hat{c}_{t-1} \hat{y}_{t}$ are not linearly independent either. Hence, stochastic singularity imposes restrictions on the moments that can be exploited for its estimation by SMM.

For the RBC model studied here, a set of linearly independent objects is $\left(\hat{y}_{t}^{2}, \hat{c}_{t}^{2}, \hat{c}_{t} \hat{y}_{t}\right.$, $\left.\hat{c}_{t} \hat{c}_{t-1}, \hat{y}_{t} \hat{y}_{t-1}\right)$. These variables are linearly independent despite relations (4) and (5) because $\hat{n}_{t} \hat{y}_{t}, \hat{y}_{t} \hat{c}_{t-1}$ and $\hat{c}_{t} \hat{y}_{t-1}$ are not included in $\mathbf{m}_{i}$. For the Monte Carlo experiments below, I also use $\mathbf{m}_{i}=\left(\hat{y}_{t}^{2}, \hat{n}_{t}^{2}, \hat{n}_{t} \hat{y}_{t}, \hat{n}_{t} \hat{n}_{t-1}, \hat{y}_{t} \hat{y}_{t-1}\right)^{\prime}$ and $\mathbf{m}_{i}=\left(\hat{n}_{t}^{2}, \hat{c}_{t}^{2}, \hat{c}_{t} \hat{n}_{t}, \hat{c}_{t} \hat{c}_{t-1}, \hat{n}_{t} \hat{n}_{t-1}\right)^{\prime}$, in order to examine the sensitivity of the results to the variables and moments employed.

Stochastic singularity has different implications for SMM and Maximum Likelihood. Estimation is limited by the number of linearly independent moments under SMM, but by the number of linearly independent variables under Maximum Likelihood. The former is a weaker restriction because it is possible to find independent moments that incorporate information about more variables than those that are linearly independent. For example, for the RBC model here, one can use independent moments that involve up to two variables for estimation by SMM, but only one observable variable for estimation by Maximum Likelihood (without measurement errors added). This means that SMM might yield more precise estimates than Maximum Likelihood, despite the fact that the former is a limited information procedure. Second, SMM requires at least as many moments as parameters are to be estimated (i.e., $p \geq q$ ) but only linearly independent objects can be included in $\mathbf{m}$. Thus, singularity might impose limits on the number of structural parameters that can be identified. 


\subsection{Generalized Method of Moments}

Consider now the case where it is possible to compute analytical expressions for the unconditional moments of the variables as a function of the structural parameters. This means that in the objective function (10), the simulation-based estimate $(1 / \tau T) \sum_{i=1}^{\tau T} \mathbf{m}_{i}(\boldsymbol{\theta})$ could be replaced with its analytical counterpart $E(\mathbf{m}(\boldsymbol{\theta}))$. Then, a Generalized Method of Moments (GMM) estimator of $\boldsymbol{\theta}$ can be obtained by minimizing the distance between the empirical moments of the data and the theoretical moments predicted by the model. This approach has been followed, among others, by Christiano and Eichenbaum (1992), Burnside, Eichenbaum, and Rebelo (1993), and Ambler, Guay, and Phaneuf (1999). GMM is also used by Braun (1994) to estimate the Euler equations of a DSGE model. Christiano and den Haan (1996) examine the effects of alternative detrending methods and estimates of the weighting matrix for the small-sample properties of GMM. Although the analytical computation of the moments can be algebraically tedious, GMM estimates are computationally and statistically more efficient than SMM.

The GMM estimator is defined by

$$
\tilde{\boldsymbol{\theta}}_{g m m}=\min _{\{\boldsymbol{\theta}\}} \mathbf{G}(\boldsymbol{\theta})^{\prime} \mathbf{W G}(\boldsymbol{\theta}),
$$

where

$$
\mathbf{G}(\boldsymbol{\theta})=(1 / T) \sum_{t=1}^{T} \mathbf{m}_{t}-E(\mathbf{m}(\boldsymbol{\theta}))
$$

and $\mathbf{W}$ is the $q \times q$ optimal weighting matrix defined in (11). Under the regularity conditions in Hansen (1982):

$$
\sqrt{T}\left(\tilde{\boldsymbol{\theta}}_{g m m}-\boldsymbol{\theta}\right) \rightarrow N\left(\mathbf{0},\left(\mathbf{D}^{\prime} \mathbf{W}^{-1} \mathbf{D}\right)^{-1}\right),
$$

where $\mathbf{D}=\partial E(\mathbf{m}(\boldsymbol{\theta})) / \partial \boldsymbol{\theta}$ is a $q \times p$ matrix assumed to be finite and of full rank. In principle, one could obtain $\partial E(\mathbf{m}(\boldsymbol{\theta})) / \partial \boldsymbol{\theta}$ analytically using the expressions for the unconditional moments $E(\mathbf{m}(\boldsymbol{\theta}))$. Note, however, that these derivatives need to be computed only once, when standard errors are calculated. ${ }^{8}$ Thus, for the Monte Carlo, I follow the simpler route of computing $\partial E(\mathbf{m}(\boldsymbol{\theta})) / \partial \boldsymbol{\theta}$ numerically prior to the calculation of the standard errors. As before, the optimal weighting matrix, $\mathbf{W}$, is computed using the Newey-West estimator with a Barlett kernel.

Comparing the asymptotic variance-covariance matrix of SMM and GMM estimates, note that they differ by the term $(1+1 / \tau)$ that premultiplies $\left(\mathbf{D}^{\prime} \mathbf{W}^{-1} \mathbf{D}\right)^{-1}$ in the former case.

\footnotetext{
${ }^{8}$ Also, one might want to compute $\partial E(\mathbf{m}(\boldsymbol{\theta})) / \partial \boldsymbol{\theta}$ analytically in order to supply an expression for the gradient in the optimization routine. As we will see below, this would make GMM even more computationally efficient than alternative estimation methods.
} 
Since $\mathbf{W}$ depends only on the data, and the simulated moments converge to the analytical ones as $\tau T \rightarrow \infty$, then the difference in the standard errors of both estimates is primarily due to the term $(1+1 / \tau)$, rather than to differences in the estimates of $\mathbf{W}$ and $\mathbf{D}$. The term $(1+1 / \tau)$ can be thought of as a measure of the increase in sample uncertainty due to the use of simulation to compute the population moments. Since $(1+1 / \tau) \rightarrow 1$ as $\tau \rightarrow \infty$, the efficiency of SMM converges to that of GMM as the length of the simulated series increases. Moreover, since $\tau$ is chosen by the researcher, the effect of simulation on sample uncertainty can be reduced by selecting an appropriately large value of $\tau$.

It is easy to see that the effect of simulation on sample uncertainty decreases rapidly as $\tau$ increases. For $\tau=5$, the standard errors of SMM estimates are $(1+1 / 5)^{1 / 2} \approx 1.10$ times larger than the ones obtained using GMM. For $\tau=10$ and 20, they are approximately 1.05 and 1.025 times larger than those obtained using GMM. Hence, the difference in statistical efficiency between SMM and GMM can be made reasonably small by increasing $\tau$. However, we will below that SMM is less computationally efficient than GMM and that the time per replication under SMM increases proportionally with $\tau$.

The implications of stochastic singularity for GMM estimation are the same as those for SMM. Singularity limits the moments that can be exploited by GMM to those that form a linearly independent set. Because identification requires at least as many moments as parameters are to be estimated, singularity might also limit the number of parameters that can be identified. Finally, GMM might deliver more precise estimates than Maximum Likelihood because one can use information on more observable endogenous variables. For example, for the RBC model in Section 2, GMM can use moments computed using up to two variables, but Maximum Likelihood (without measurement errors) can only use one observable variable.

A global specification test for overidentified DSGE models can be performed using the chi-square statistic

$$
T\left[\mathbf{G}\left(\tilde{\boldsymbol{\theta}}_{g m m}\right)^{\prime} \mathbf{W G}\left(\tilde{\boldsymbol{\theta}}_{g m m}\right)\right] \rightarrow \chi^{2}(p-q),
$$

where $\mathbf{G}\left(\tilde{\boldsymbol{\theta}}_{\text {gmm }}\right)^{\prime} \mathbf{W} \mathbf{G}\left(\tilde{\boldsymbol{\theta}}_{\text {gmm }}\right)$ is the value of the objective function at the optimum.

\subsection{Indirect Inference}

This section examines the Indirect Inference procedure proposed by Smith (1993). For reasons to be made clear below, Smith refers to this procedure as Extended Method of Simulated Moments (EMSM). In order to understand the mechanics of EMSM, it is useful to recall that SMM constructs an estimate of $\boldsymbol{\theta}$ by minimizing the distance between the unconditional moments of the data and those of an artificial series simulated using given 
parameter values. In contrast, EMSM constructs an estimate of $\boldsymbol{\theta}$ by minimizing the distance between the parameters of a Vector Autoregression (VAR) estimated from the data and those estimated from an artificial series simulated using given parameter values. This approach has a Method of Moments interpretation because the coefficients of the VAR are proportional to covariances and autocovariances of the series in the VAR. This approach also has an Indirect Inference interpretation [Gouriéroux, Monfort, and Renault (1993)] because the auxiliary model (the VAR) is a misspecified version of the true state-space representation of the model. ${ }^{9}$

More formally, denote by $\boldsymbol{\eta}$ the $p \times 1$ vector with the estimates of a VAR representation of the data. Denote by $\boldsymbol{\eta}(\boldsymbol{\theta})$ the synthetic counterpart of $\boldsymbol{\eta}$ with the estimates of a VAR representation of artificial data generated by the DSGE model. As before, the sample size is denoted by $T$ and the number of observations in the artificial time series is $\tau T$. Then, the Indirect Inference estimator of $\boldsymbol{\theta}, \tilde{\boldsymbol{\theta}}_{i m}$, is the value that solves

$$
\min _{\{\boldsymbol{\theta}\}}[\boldsymbol{\eta}-\boldsymbol{\eta}(\boldsymbol{\theta})]^{\prime} \mathbf{V}[\boldsymbol{\eta}-\boldsymbol{\eta}(\boldsymbol{\theta})],
$$

where $\mathbf{V}$ is the $p \times p$ optimal weighting matrix. In the case where the information matrix equality holds, Smith suggests using the inverse of the variance-covariance matrix of the estimate $\boldsymbol{\eta}$ as an estimator of $\mathbf{V}$.

Under the regularity conditions in Smith (1993),

$$
\sqrt{T}\left(\tilde{\boldsymbol{\theta}}_{i m}-\boldsymbol{\theta}\right) \rightarrow N\left(\mathbf{0},(1+1 / \tau)\left(\mathbf{J}^{\prime} \mathbf{V}^{-1} \mathbf{J}\right)^{-1}\right),
$$

where $\mathbf{J}=E(\partial \boldsymbol{\eta}(\boldsymbol{\theta}) / \partial \boldsymbol{\theta})$ is a $q \times p$ matrix assumed to be finite and of full rank. In the Monte Carlo, the derivatives $\partial \boldsymbol{\eta}_{t}(\boldsymbol{\theta}) / \partial \boldsymbol{\theta}$ are computed numerically and the expectation approximated by the average over the simulated $\tau T$ data points. Smith suggests a test based on Hansen's (1982) chi-square statistic as specification test of an overidentified DSGE model:

$$
T(1+1 / \tau)\left\{\left[\boldsymbol{\eta}-\boldsymbol{\eta}\left(\tilde{\boldsymbol{\theta}}_{i m}\right)\right]^{\prime} \mathbf{V}\left[\boldsymbol{\eta}-\boldsymbol{\eta}\left(\tilde{\boldsymbol{\theta}}_{i m}\right)\right]\right\} \rightarrow \chi^{2}(p-q),
$$

where $\left[\boldsymbol{\eta}-\boldsymbol{\eta}\left(\tilde{\boldsymbol{\theta}}_{i m}\right)\right]^{\prime} \mathbf{V}\left[\boldsymbol{\eta}-\boldsymbol{\eta}\left(\tilde{\boldsymbol{\theta}}_{i m}\right)\right]$ is the value of the objective function at the optimum.

The singularity of the DSGE model has implications for both the order and the number of variables included in the auxiliary VAR. More precisely, the artificial data generated by the DSGE model does not have an unconstrained VAR representation. In the case of the

\footnotetext{
${ }^{9}$ Rather than matching VAR parameters, some authors match instead the impulse-responses of the model. Since the impulse-responses are nonlinear transformations of the VAR parameters, this strategy is similar to the one studied here. For an application of this idea, see Christiano, Eichenbaum, and Evans (2001). An advantage of matching impulse responses is that the researcher can focus on specific horizons by a choosing an appropriate weighting matrix.
} 
RBC model studied here, recall that the systems $\left(\hat{n}_{t}, \hat{y}_{t}, \hat{c}_{t}\right),\left(\hat{y}_{t}, \hat{c}_{t}, \hat{y}_{t-1}, \hat{c}_{t-1}\right),\left(\hat{y}_{t}, \hat{n}_{t}, \hat{y}_{t-1}\right.$, $\left.\hat{n}_{t-1}\right)$, and $\left(\hat{n}_{t}, \hat{c}_{t}, \hat{n}_{t-1}, \hat{c}_{t-1}\right)$ are singular, meaning that their sample variance-covariance matrices are singular. This implies that the data generated by this simple DSGE model has only a bivariate VAR representation of order one. ${ }^{10}$ Any attempt to estimate a VAR with the three observable variables and/or using more than one lag will fail because the matrix of explanatory variables is not of full rank.

Since in constructing the synthetic VAR, one is limited to two of the three observable endogenous variables and to only one of their lags, I use VARs of order one on $\left(\hat{y}_{t}, \hat{n}_{t}\right),\left(\hat{y}_{t}\right.$, $\left.\hat{c}_{t}\right)$ and $\left(\hat{n}_{t}, \hat{c}_{t}\right)$ in the Monte Carlo experiments below in order to examine the sensitivity of the results to the variables used.

\section{Monte-Carlo Experiments}

\subsection{Design}

The small-sample properties of all estimators are studied here using Monte Carlo analysis. First, I study the small-sample properties under the null hypothesis. That is, the Data Generating Process (DGP) is the linearized, one-sector RBC model with indivisible labor and one structural (technology) shock that was described in Section 2. Second, I study the small-sample properties in the case where the DSGE model is misspecified. In this case the DGP is also a linearized, one-sector RBC model with indivisible labor, but with three structural shocks. In addition to the technology shock, the model incorporates a preference shock and a shock to the investment function.

All experiments are based on 500 replications using a sample size of 200 observations. This sample size corresponds to, say quarterly observations of the series for a period of 50 years. In order to limit the effect of the starting values used to generate the series, 100 extra observations were generated in every replication. Then, for the estimation of the model, the initial 100 observations were discarded.

I focus on three observable variables, namely output, consumption and hours worked, and examine whether estimates are sensitive to using different combinations of these variables in the estimation procedures. In order to reduce the computational burden in the Monte Carlo experiments, I concentrate on three (of the five) model parameters, namely the subjective discount factor $(\beta)$, the autocorrelation coefficient of the technology shock $(\rho)$, and the

\footnotetext{
${ }^{10} \mathrm{Smith}$ is able to specify a bivariate VAR of order two for a model similar to the one here because he assumes a second disturbance that affects the productivity of the new investment good. Since in this case, the linearized law of motion for capital has an error term, systems like $\left(\hat{y}_{t}, \hat{c}_{t}, \hat{y}_{t-1}, \hat{c}_{t-1}\right)$ in $(5)$ are not singular.
} 
standard deviation of the technology innovation $(\sigma)$. Thus, $\boldsymbol{\theta}=(\beta, \rho, \sigma)^{\prime}$ is a $3 \times 1$ vector. The data were generated using parameter values $(\beta, \rho, \sigma)=(.95, .85, .04)$. The share of capital in production $(\alpha)$ and the depreciation rate $(\delta)$ were fixed in all experiments to .36 and .025, respectively. This Monte Carlo design is realist in the sense that the true parameter values correspond to the ones typically found in empirical analysis. ${ }^{11}$

For the Monte Carlo experiments with priors, I consider independent prior densities for $\beta$ and $\rho: \beta \sim N\left(.95, .025^{2}\right), \rho \sim N\left(.85, .07^{2}\right)$, and a diffuse prior for $\sigma$. In terms of the prior representation in $(8)$, they correspond to

$$
\left[\begin{array}{l}
.95 \\
.85
\end{array}\right]=\left[\begin{array}{ll}
1 & 0 \\
0 & 1
\end{array}\right]\left[\begin{array}{l}
\beta \\
\rho
\end{array}\right]+\mathbf{e}, \text { with } \mathbf{e} \sim N\left(\left[\begin{array}{l}
0 \\
0
\end{array}\right],\left[\begin{array}{cc}
.025^{2} & 0 \\
0 & .07^{2}
\end{array}\right]\right) .
$$

The priors for $\beta$ and $\rho$ mean that, before observing the data, the researcher believes that with a 95 per cent probability their true values are in the intervals $(.901, .999)$ and $(.713, .987)$, respectively.

For SMM and Indirect Inference, I used three different values of $\tau=5,10,20$, meaning that the simulated series are, respectively, 5, 10, and 20 times larger than the sample size of 200 observations. Here, I also simulated 100 extra observations in every simulation and then discarded the initial 100 observations when computing the moments or the VAR parameters. For these simulation procedures, I fixed the seed in the random numbers generator in each replication and used the same draw for the model estimation. A problem with using blocks of random numbers is that they are perforce small [Ripley (1987, p. 138)]. However, in this case the use of common random draws is essential to calculate the numerical derivatives of the maximization algorithm. Otherwise, the objective function would be discontinuous and the optimization algorithm would be unable to distinguish a change in the objective function due to a changes in the parameters from a change in the random draw used to simulate the series.

\subsection{Results under the Null Hypothesis}

Monte Carlo results are reported in Tables 1 to 3 . In all tables, Mean is the average of the estimated parameter values. A.S.E. is the average asymptotic standard error. Both averages are taken over the 500 replications in each experiment. Median and S.D. are the median and standard deviation of the empirical parameter distribution. Comparing Median

\footnotetext{
${ }^{11}$ A slightly larger value of $\beta$, say $\beta=.99$, would more appropriate if one were to adhere to the interpretation of the series as quarterly observations of the variables. However, from the numerical perspective, it is convenient to work with a value of $\beta$ that is close, but not too close, to the admissible boundary of 1 . In unreported work, I performed a very limited set of Monte-Carlo experiments using the parameterization $\left(\beta, \rho, \sigma_{\epsilon}\right)=(.98, .95, .04)$ with similar results to the ones reported.
} 
and Mean with the true parameter value, and S.D. with A.S.E., is informative about the small-sample distribution of the estimates. For example, if the Mean is well below the true parameter value, this indicates the downward bias of the estimate. If Mean and Median are substantially different, this indicates that the small-sample distribution of the estimates is skewed. If S.D. is much larger than A.S.E., this indicates that using the asymptotic formula to compute the standard error might understate the true variability of the estimate in small samples.

In all tables, Size is the proportion of times that the null hypothesis that the parameter takes its true value is rejected using a $t$-test with nominal size of 5 per cent. In other words, Size denotes the empirical size of this $t$-test. S.E. is the standard error of this empirical size and is computed as the standard deviation of a Bernoulli variable. In ideal circumstances, the nominal and empirical sizes of the $t$-test would be close. More formally, the 95 per cent confidence interval around the empirical size would contain the nominal size of 5 per cent. However, we will see below that in practice there are substantial size distortions because the asymptotic standard error is not always a good approximation to the small-sample standard error of the estimates. In Tables 2 and 3, OI is the empirical size of the $\chi^{2}$ test of the overidentification restrictions.

Panel A in Table 1 reports results using Maximum Likelihood without adding measurement errors/priors. Recall that singularity implies that the RBC model cannot be estimated by Maximum Likelihood using more than one observable variable. Experiments 1 through 3 refer to the experiments using output, consumption, and hours worked, respectively.

Despite the fact that the model is a general equilibrium one, not all variables appear to be equally informative about all structural parameters. In other words, the choice of variable(s) employed in Maximum Likelihood estimation might matter. We will see below that this is also true for the Methods of Moments. In particular, the average estimate and A.S.E. of $\beta$ vary substantially depending on whether one uses data on output, consumption, or hours worked. When the model is estimated using data on output alone, the average estimate of $\beta$ is well below its true value and the A.S.E. is very large. When the model is estimated using data on either consumption or hours worked, the average estimate is close to the true value and the A.S.E. is small. All this means that for the simple RBC model studied here, a sharper estimate of the subjective discount factor can be obtained using consumption or hours worked, rather than output. The effect of the choice of variable on the point estimates of the other structural parameters is minor. However, the standard error of the empirical distribution and the A.S.E. of $\rho$ seem to vary with the variable employed. For example, the A.S.E. of $\rho$ is much larger when consumption, rather than output, is used to estimate the model. Thus, the autoregressive coefficient of the technology shock is estimated more 
precisely using output than either consumption or hours worked.

The asymptotic standard errors approximate well the small-sample standard deviation of the estimates. However, the difference between them is large enough that in some cases there are size distortions for the $t$-test of the null hypothesis that the true parameter value is the one used to generate the data.

Panel B in Table 1 reports results using Maximum Likelihood incorporating the priors specified in (14). Experiments 4 through 6 refer, respectively, to the experiments using output, consumption, and hours worked. Because the prior about $\sigma$ was uninformative, results regarding this parameter are basically the same as those reported in Panel A. Regarding $\beta$ and $\rho$, the mean of the prior density was, by design, the same as the true value used to generate the sample. Recall that an interpretation of the prior is that of a penalty on the log likelihood function as the estimate deviates from the prior mean. As a result, estimates obtained using Maximum Likelihood with priors tend to be numerically closer to their true values than ML estimates without priors. An interesting case is the estimate of $\beta$ obtained using output data. Results from Panel A suggest that output is not very informative regarding the discount factor. Consequently when the prior and the output data are combined, the resulting posterior distribution of the estimate looks very similar to the prior density.

In most cases, the A.S.E. is larger than the standard deviation of the parameter estimates. Hence, asymptotic standard errors overestimate the variability of the parameter estimates in small samples. As a result, the $t$-test of the hypothesis that the parameter takes its true value has a smaller empirical than nominal size and tends to underreject the null hypothesis. ${ }^{12}$

Table 2 report results using the Simulated and Generalized Methods of Moments. In all cases, the mean and median of the estimated parameters are close to their true values. Standard errors are reasonably low and vary with $\tau$ as predicted by the discussion in Section 3.4. ${ }^{13}$ That is, SMM standard errors based on simulated series with $\tau=5,10$ and 20 are roughly 1.1,1.05, and 1.025 times larger than those obtained using GMM, respectively.

\footnotetext{
${ }^{12}$ Notice that when the DGP is the RBC model with one shock, it is not possible to estimate the model by Maximum Likelihood with measurement errors added. The reason is that under the null, the mean and variance of measurement errors is zero. Results using ML with measurement errors are reported below for the case where the model is misspecified. The previous version of this paper [Ruge-Murcia (2002, Table $2)$ ] reports results where the DGP is the one-shock model with extra errors added. Those results are not comparable with the ones reported here because the DGP is not the same

${ }^{13}$ Recent research [see, for example, Fuhrer, Moore, and Schuh (1995)] shows that GMM can yield biased parameter estimates when applied to conditional moments of the data (e.g., Euler equations). The reason is that in this case instruments are used to frame the model implications in terms of orthogonality conditions to which GMM is then applied. Their research finds that GMM can have very poor small sample properties when the instruments are weak. However, notice that GMM is applied here to unconditional moments of the data and no instruments are required. This means that the problems caused by weak instruments do arise here, but could arise potentially when one combines conditional and unconditional moments to estimate DSGE models by GMM.
} 
However, this variation in efficiency is of the same order of magnitude as that observed across moments employed in the estimation procedures. This means that the choice of the length of the simulated series in SMM might be as important as the choice of moments to match.

As reported above for Maximum Likelihood, the choice of moments to match under SMM and GMM can have some effect on the precision of the parameter estimates. This is because not all moments are equally informative about all structural parameters. For example, in Panel $\mathrm{A}$, the standard deviation of the estimate of $\beta$ obtained using the moments of output and hours worked is roughly 30 per cent larger than the one obtained using the moments of consumption and hours worked, for the same value of $\tau$.

There are some differences between the A.S.E. and the standard deviations of the empirical distribution of the parameters. Since the $t$-statistics are computed using asymptotic standard errors, this translates into fairly large size distortions for the $t$-test. There is no obvious pattern for the size distortions, but they appear to vary more with the moments matched than with the length of the simulated series (in SMM).

The last column in Table 2 report the empirical size of the $\chi^{2}$ test of the overidentification restrictions of the model. Notice that in all cases the empirical size of the test is well below its nominal size of 5 per cent. This means that a researcher comparing the $\chi^{2}$ statistic with the 5 per cent critical value of the appropriate distribution is very likely to conclude that the overidentification restrictions of the DSGE model cannot be rejected. This is because rather than taking a 5 per cent probability of rejecting a true model, the researcher actually takes approximately a 1 per cent probability. In some cases (see, for example, Experiment 9 in Panel C), the model's overidentification restrictions were never rejected by the $\chi^{2}$ test in the 500 replications. The fact that Hansen's $\chi^{2}$ easily fails to detect a misspecified model is well known in the literature [see, among others, Newey (1985)]. The results in this paper suggest that the severe size distortions of the $\chi^{2}$ test also arise in the context of fully-specified DSGE models.

Finally, Table 3 reports results using Indirect Inference. In all experiments, the mean and median of the estimated parameters are close to their true values. The standard errors of the estimates of $\beta$ and $\rho$ are large, but the standard deviation of the innovation to the technology shock, $\sigma$, is estimated more precisely by Indirect Inference than by any other method. As in Maximum Likelihood, SMM, and GMM, the choice of variables/moments to use in estimation has some effect on the precision of the estimates. This can be traced back to the fact that not all variables/moments are equally informative about all structural parameters. For example, $\sigma$ can only be identified under Indirect Inference when $\boldsymbol{\eta}$ includes the standard deviation of the residuals of the Vector Autoregression. Thus, the autocorrelations and cross correlations of the variables do not seem informative regarding the standard deviation of technology 
innovations. In line with the finding that under Maximum Likelihood, consumption and hours worked are more informative than output about the subjective discount rate, the standard deviation of the empirical distribution of $\beta$ is smallest when the VAR consist of $\hat{c}_{t}$ and $\hat{n}_{t}$. There are very large size distortions for both the $t$-test that the parameters take their true value and the $\chi^{2}$ test of the overidentification restrictions. As in the Methods of Moments, there is no clear pattern for these size distortions, but they seem to vary more with the moments matched than with the length of the simulated series.

It is enlightening to go beyond the summary statistics in these Tables and plot the empirical distribution of the parameter estimates obtained under the different methods. The frequency histograms for estimators of $\beta, \rho$, and $\sigma$ are plotted in Figures 1 to 3, respectively. They correspond to results in Experiments 3 and 6 in Table 1, Experiments 5, 6, 11, and 12 in Table 2, and Experiments 5 and 6 in Table 3. The reason I focus on these experiments, is because they help illustrate more general results uncovered by the Monte Carlo analysis.

Four conclusion can be drawn from these Figures. First, the Methods of Moments estimators can deliver more precise estimates than Maximum Likelihood. This was anticipated from the earlier observation that singularity implies that the RBC model can be estimated by Maximum Likelihood using only one observable variable, but using moments of up to two variables by the Methods of Moments. However, it is very likely that this finding is specific to the one-shock RBC model examined here and might not carry over to the models with a larger number of structural shocks. For example, in the preliminary work mentioned in footnote 2, where there were two structural shocks, Maximum Likelihood and SMM performed equally well.

Second, informative priors can be combined with sample data to sharpen the researcher's inferences regarding the structural parameters of DSGE model. This can be seen in Figures 1 and 2 by comparing the empirical distribution of the estimators of $\beta$ and $\rho$ obtained using Maximum Likelihood with and without priors.

Third, the difference in efficiency between GMM and SMM does not appear to be very large, though the empirical distribution obtained by SMM with $\tau=5$ (not reported) is somewhat more diffuse than the others. Hence, the effect of simulation on sample uncertainty and the precision of parameter estimates can be moderated by a suitable choice of $\tau$.

Fourth, the choice of variables/moments employed can have some effect on the precision of the estimators. This can be seen in Figures 1 and 2 by comparing the empirical distribution of the estimators of $\beta$ and $\rho$ obtained by SMM, GMM, and Indirect Inference using the moments of output/hours worked and consumption/hours worked. Indirect Inference estimates based on the moments of output/hours worked have a very diffuse empirical distribution and a substantial number of outliers, though their mean is close to the true parameter 
value used to generate the sample.

One of the reason DSGE model are interesting is because they allow the researcher to examine the response of a model economy to shocks. An advantage of the full estimation of DSGE models is that parameter uncertainty can be incorporated to construct confidence intervals around the model's dynamic response to a shock. Since impulse-responses depend nonlinearly on the structural parameters, it is useful to examine how the precision of the estimates translates into less or more precise impulse-responses. Figure 4 plots the dynamic responses of consumption, output, and hours worked following a technology shock. The dotted lines are the 90 per cent confidence intervals for the response. The parameter estimates used to construct these figures come from the same experiments used to obtain Figures 1 to 3 .

Figure 4 supports four conclusions. First, the mean response does not depend on the estimation method because all methods deliver unbiased parameter estimates. However, there are some small differences in the coverage probabilities of the estimated confidence intervals because not all estimation procedures are equally efficient. Second, confidence intervals for the response based on Indirect Inference estimates obtained using output/hours worked moments are the widest. This reflects the diffuse empirical distributions of $\beta$ and $\rho$ reported in Figures 1 and 2. Third, aside from the latter case, there are no large differences in the confidence intervals obtained using different estimators of the structural parameters. This means that the differences in parameter efficiency across estimation methods reported above, does not translate necessarily into substantial differences in the coverage probabilities of their impulse responses.

\subsection{Robustness to Misspecification}

This section report results of Monte Carlo experiments where the DSGE model is misspecified. The intention here is to study the robustness of the different estimation methods to misspecification. To that end, the artificial data is generated by the linearized, one-sector RBC model with indivisible labor, but with three (rather than only one) structural shock. It is assumed that, in addition to the technology shock, the true model incorporates a preference shock and a shock to the investment function. The preference shock is a disturbance to the weight of leisure in the utility function, $\psi$. This weight follows the stochastic process

$$
\ln \psi_{t+1}=0.6 \ln \psi_{t}+\zeta_{t},
$$

where $\zeta_{t}$ is an innovation assumed to be independently, identically, and Normally distributed with zero mean and variance $0.03^{2}$. The shock to the investment function affects the transformation of investment into new capital. In particular, the law of motion of the capital 
stock is generalized to:

$$
k_{t+1}=(1-\delta) k_{t}+\nu_{t} x_{t}
$$

where $\nu_{t}$ is a shock that follows the process

$$
\ln \nu_{t+1}=0.6 \ln \nu_{t}+\varepsilon_{t},
$$

where $\varepsilon_{t}$ is an innovation assumed to be independently, identically, and Normally distributed with zero mean and variance $0.04^{2}$. Notice that the mean of $\nu_{t}$ equals 1 so that on average one unit invested becomes one unit of capital. Since there are now three structural shocks, a researcher looking at the (artificial) data series on, say output, consumption, and hours worked would find them to be nonsingular. The estimated model is the economicallyinteresting but misspecified and stochastically-singular RBC model with only one technology shock.

Results are reported in Tables 4 to 6 . In all tables, Bias is the average difference between the estimated parameter values and the true value used to generate the artificial data. Remaining headings are a previously described. Table 4 reports results obtained using Maximum Likelihood. Panel A considers the case where neither priors nor measurement errors are used in estimation, and the researcher is limited to using at most as many variables as structural shocks. Estimates of $\sigma$ are biased upwards, while estimates of $\beta$ and $\rho$ are biased downwards. The biases are more severe when data on hours worked or output, rather than consumption, is used. The reason $\sigma$ is biased upwards is that all the conditional variance of the series is attributed to the technology shock.

In order to understand why different series lead to different estimates, recall that Maximum Likelihood minimizes the one-step-ahead predictions of the model. The variance decomposition of the true model with multiple shocks indicates that the technology shock explains 89, 78, and 44 per cent of the conditional variance of the one-step ahead forecasts errors of consumption, output, and hours worked, respectively. Because the only shock in the estimated RBC model (that is, the technology shock) is more important in explaining the variation of consumption in the short-run than the one of hours worked or output, the misspecification affects ML estimates less severely when one uses data on consumption than hours worked or output.

Note also in Panel A, that the asymptotic standard errors usually underestimate the standard errors of the empirical, small-sample distribution of the estimates. Consequently, $t$-tests tend to reject the null hypothesis that the parameters take their true value more often that the nominal size of 5 per cent.

Panel B in Table 4 reports results using Maximum Likelihood but incorporating Bayesian priors. In general, using priors reduces the bias of the estimates and the variance of their 
small-sample distributions. One of the reasons because of which biases are reduced is simply that the priors are centered around the true parameter value. More generally, incorporating prior information will "pull" ML estimates towards more economically reasonable values, even if the estimated model is misspecified. The asymptotic standard errors tend to overestimate the standard error of the small-sample distribution and, as a result, $t$-tests tend to reject the null hypothesis less often than 5 per cent of the times.

Finally, Panel C reports the results using Maximum Likelihood but adding measurement errors. Because measurement errors are added, more variables than the number of structural shocks can be used in Maximum Likelihood estimation. In general, the bias of the estimates are smaller in this case than the ones reported in Panel A. However, because the technology shock is not equally important in explaining the short-run behavior of all series, results depend on the data used in estimation. For the example considered here, biases are less severe for combinations of variables that include consumption. Finally, note that, as in Panel A, the asymptotic standard errors underestimate the standard errors of the smallsample distribution of the estimates. This means that the $t$-test that the parameters take their true value will overreject.

Table 5 report results for the Simulated and Generalized Method of Moments. Estimate of $\rho$ are downward biased in all cases but, in general, the bias of the Methods of Moments estimates is smaller than that of ML estimates. Because the asymptotic standard errors differ from the small-sample standard errors, the $t$-test is subject to size distortions, but these distortions are smaller than the ones observed in Table 4 for Maximum Likelihood. Hence, the Methods of Moments appear more robust than Maximum Likelihood to the form of misspecification considered here, in the sense that biases are smaller and statistical inference is less affected.

Finally note that, as in Table 2, standard errors vary with $\tau$ as a result of the uncertainty introduced by simulation error. As predicted by the discussion in Section 3.4, SMM standard errors based on simulated series with $\tau=5,10$ and 20 are roughly 1.1,1.05, and 1.025 times larger than those obtained using GMM, respectively. However, this variation in efficiency is much smaller than that observed across moments employed in estimation. Thus, when the model is misspecified, the choice of moments to match might be more important than the length of the simulated series in SMM.

Table 6 reports results using Indirect Inference. In general, results are similar to those obtained the Methods of Moments. The reason is that the version of Indirect Inference studied here matches coefficients on a VAR representation of the data and these coefficients are proportional to covariances and autocovariances of the series in the VAR. These explains why the bias in the parameter estimates are usually smaller than those observed under Maxi- 
mum Likelihood and roughly of the same magnitude as those obtained under the Methods of Moments. However, the size distortions of the $t$-test are usually larger than those observed under the Methods of Moments and there are instances where the bias of $\hat{\beta}$ and $\hat{\rho}$ is severe.

Figure 5 compares the biases in all experiments under each estimation method. In particular, I focus on the bias of the estimates of $\beta$ and $\rho$ by plotting them in a plane with the bias of $\tilde{\beta}$ is in the horizontal axis and that of $\tilde{\rho}$ is in the vertical axis. Notice that for the Methods of Moments estimators, the biases are closely packed near the $(0,0)$ point, meaning that, although not exactly zero, these biases are comparatively small. For Indirect Inference, that is also true in most cases, but there are some outliers where the biases are large. By far the most diffuse plot is that of Maximum Likelihood. The points closest to $(0,0)$ are those where Bayesian priors were incorporated in estimation. Biases tend to be generally larger than under the Methods of Moments, and there are cases where they can be very severe.

Figure 6 examines the effects of misspecification and bias on impulse response analysis. In particular, I compare the response to a technology shock for the misspecified model (continuous line) with the response predicted by the true model (dotted line). These responses were constructed using estimates from Experiments 3, 6, and 9 in Table 3, Experiments 5, 6, and 12 in Table 4, and Experiments 5 and 6 in Table 6 . In order not to saturate the picture, I have abstained from plotting confidence intervals of these responses.

Consider first the responses predicted by GMM and SMM estimates in columns 4 to 6 . Quantitatively, the (average) estimated responses are not too far from the true response and the predicted dynamics are roughly similar. Statistically, the true response lies just above the 90 per cent confidence bound (not shown). Note that because, GMM and SMM results in Table 5 are similar, these plots are representative of the effect of misspecification on impulse response analysis with GMM and SMM estimates.

Columns 1 to 3 plot the responses obtained using ML estimates. When no priors are added, the (average) estimated response can be quite different from the true response, both in terms of numerical and statistical distance and in terms of the dynamics. Notice, however, that these results are not representative of all ML results. For example, responses using results from Maximum Likelihood estimation with consumption data (not shown) yield responses whose 90 per cent confidence interval includes the true response. Still, these plots illustrate the general observation that Maximum Likelihood is generally less robust to the form of misspecification considered here than the Methods of Moments.

Columns 7 and 8 plot the responses obtained using Indirect Inference estimates. These plots illustrate the previous observation that matching VAR coefficient will lead to similar results that matching moments directly. However, the moments coded in the VAR might 
not always be the most informative. In this case, biases can be severe and predicted impulse response might differ substantially from the true response.

In general, results from these two sections are in line with the general econometric observations that 1) when the model is correctly specified Maximum Likelihood is more efficient than limited information procedures, like the Methods of Moments, but 2) when the model is misspecified, limited information procedures are more robust. In the case of DSGE models, the first observation is qualified by the fact that stochastic singularity affects Maximum Likelihood more severely than the Methods of Moments. Consequently, both Maximum Likelihood and the Methods of Moments might be similarly efficient when the DSGE model is correctly specified.

\subsection{Comparison in Terms of Computing Time}

The estimation of DSGE models can be computationally demanding because the model needs to be solved for each observation in each iteration of the optimization procedure that maximizes (or minimizes) the relevant objective function. Thus, an important goal of this paper is to compare the different estimation methods in terms of their computing time. Table 7 reports in the first column the average number of seconds taken to complete a replication, including the computation of standard errors. The average is taken over all replications for all experiments in Tables 1 to 3 that employ the same estimation method. The second column reports the ratio of the number in column one to the corresponding one for GMM, that is taken as benchmark. For example, for Maximum Likelihood this ratio is 3.44/.67 $=5.1$, and means that Maximum Likelihood takes on average 5 times longer than GMM to complete a replication.

From this Table is clear that GMM is by far the most efficient procedure computationally, followed by Maximum Likelihood. On the other hand, GMM requires the analytical calculation of the unconditional moments implied by the model. This task can be algebraically tedious and time-consuming for models more complicated than the one studied here. There is a large difference in computational efficiency between SMM/Indirect Inference and GMM, and the time per iteration appears to increase proportionally with $\tau$. The reason is that SMM and Indirect Inference require the solution of the DSGE model and computation of the gradients using $\tau$ times more observations than GMM. ${ }^{14}$

\footnotetext{
${ }^{14}$ Results regarding EMSM need to be interpreted with caution. For all estimation methods, the maximization (or minimization) routines were started at the true parameter values in order the make the Monte Carlo experiment more efficient. However, I found that for EMSM, the algorithm would frequently blow up if the routine was started at the true value of $\sigma$. Hence, for EMSM, the minimization routine was started using a value for $\sigma$ much larger than the one used to generate the sample. Just for this reason alone EMSM would take longer to converge than the other estimation methods. This means that the numbers in Table
} 


\section{Discussion}

The paper studies the application of standard econometric techniques for the estimation of Dynamic Stochastic General Equilibrium models. Although stochastic singularity imposes restrictions on the variables/moments that can be used in estimation and affects more severely Maximum Likelihood than the Methods of Moments, all procedures yield unbiased and reasonably precise parameter estimates under the null hypothesis. Monte Carlo results indicate that, despite the fact that the model is a general equilibrium one, not all variables/moments are equally informative about all structural parameters. Since all procedures deliver consistent estimates, this has only a small effect on the point estimates but it appears to have some moderate implications for the small-sample standard errors.

For the one sector Real Business Cycle model studied here, the Methods of Moments deliver more precise estimates than Maximum Likelihood without added measurement errors. However, this finding is probably specific to one-shock DSGE models and might not carry on to the models with a larger number of structural shocks that are more representative of the current state of the literature. Although the Simulated and Generalized Methods of Moments are roughly comparable in terms of statistical efficiency, SMM is more computationally demanding.

When the model is misspecified, Monte-Carlo results are in line with the general econometric observations that limited information procedures are more robust than Maximum Likelihood. However, incorporating Bayesian priors "pulls" ML estimates towards more economically reasonable values and reduces both the bias of the estimates and the variance of their small-sample distributions.

7 most likely overstate the computating time required by EMSM. 
Table 1. Maximum Likelihood Results

Under the Null Hypothesis

(Artificial Data is Stochastically Singular)

\begin{tabular}{|c|c|c|c|c|c|c|c|c|c|c|}
\hline \multirow{2}{*}{\multicolumn{2}{|c|}{ Experiment }} & \multicolumn{3}{|c|}{$\beta$} & \multicolumn{3}{|c|}{$\rho$} & \multicolumn{3}{|c|}{$\sigma$} \\
\hline & & $\begin{array}{l}\text { Mean } \\
\text { Median }\end{array}$ & $\begin{array}{l}\text { A.S.E. } \\
\text { S.D. }\end{array}$ & $\begin{array}{l}\text { Size } \\
\text { S.E. }\end{array}$ & $\begin{array}{l}\text { Mean } \\
\text { Median }\end{array}$ & $\begin{array}{l}\text { A.S.E. } \\
\text { S.D. }\end{array}$ & $\begin{array}{l}\text { Size } \\
\text { S.E. }\end{array}$ & $\begin{array}{l}\text { Mean } \\
\text { Median }\end{array}$ & $\begin{array}{c}\text { A.S.E. } \\
\text { S.D. }\end{array}$ & $\begin{array}{l}\text { Size } \\
\text { S.E. }\end{array}$ \\
\hline \multicolumn{11}{|c|}{ A. Using as Many Variables as Shocks } \\
\hline \multirow[t]{2}{*}{1} & $\hat{y}_{t}$ & .8045 & .2218 & .0740 & .8114 & .0595 & .0360 & .0487 & 0181 & .2120 \\
\hline & & .8430 & 1688 & 0117 & .8218 & 0586 & .0083 & .0488 & 0102 & .0183 \\
\hline \multirow[t]{2}{*}{2} & $\hat{c}_{t}$ & .9256 & .0859 & 1440 & .8203 & 1229 & .0580 & 0511 & .0366 & .0860 \\
\hline & & .9462 & 1038 & .0157 & .8562 & .1172 & .0105 & .0375 & .0328 & .0125 \\
\hline \multirow[t]{2}{*}{3} & $\hat{n}_{t}$ & .9375 & .0610 & .1420 & .8426 & .0610 & .0460 & .0440 & .0217 & 1600 \\
\hline & & .9383 & .0520 & .0156 & .8579 & .0520 & .0094 & .0444 & .0166 & .0164 \\
\hline \multicolumn{11}{|c|}{ B. Incorporating Bayesian Priors } \\
\hline \multirow[t]{2}{*}{4} & $\hat{y}_{t}$ & .9501 & .0249 & .0000 & .8425 & .0335 & .0220 & .0398 & .0037 & .0020 \\
\hline & & .9501 & .0009 & .0000 & .8446 & .0288 & .0066 & .0398 & .0022 & .0020 \\
\hline \multirow[t]{2}{*}{5} & $\hat{c}_{t}$ & .9488 & .0228 & .0000 & .8463 & .0523 & .0000 & .0396 & .0086 & .0000 \\
\hline & & .9394 & .0068 & .0000 & .8454 & .0295 & .0000 & .0396 & .0023. & .0000. \\
\hline \multirow[t]{2}{*}{6} & $\hat{n}_{t}$ & .9488 & .0222 & .0000 & .8428 & .0433. & .0020 & .0399. & .0080. & .0000. \\
\hline & & .9486 & .0061. & .0000 & 8445 & .0255 & .0020. & .0400 & .0024. & . 0000. \\
\hline
\end{tabular}

Notes: The true values are $\beta=.95, \rho=.85$, and $\sigma=.04$. Mean is the arithmetic average of the estimated parameter values; A.S.E. is the average asymptotic standard error; Median and S.D. are the median and standard deviation of the empirical parameter distribution; Size is an estimate of the actual size of the $t$-test with nominal size of 5 per cent of the null hypothesis that the parameter takes its true value; and S.E. is the standard error of the actual test size. The experiments were based on 500 replications. The priors used are: $\beta \sim N\left(.95, .025^{2}\right), \rho \sim N\left(.85, .07^{2}\right)$. The prior for $\sigma$ is diffuse. 
Table 2. Results using Methods of Moments

Under the Null Hypothesis

(Artificial Data is Stochastically Singular)

\begin{tabular}{|c|c|c|c|c|c|c|c|c|c|c|c|}
\hline \multirow{2}{*}{\multicolumn{2}{|c|}{ Experiment }} & \multicolumn{3}{|c|}{$\beta$} & \multicolumn{3}{|c|}{$\rho$} & \multicolumn{3}{|c|}{$\sigma$} & \multirow{3}{*}{$\begin{array}{r}\text { OI } \\
\text { S.E. }\end{array}$} \\
\hline & & Mean & A.S.E. & Size & Mean & A.S.E. & Size & Mean & A.S.E. & Size & \\
\hline$\#$ & Var. & Median & S.D. & S.E. & Median & S.D. & S.E. & Median & S.D. & S.E. & \\
\hline \multicolumn{12}{|c|}{ A. Simulated Method of Moments with $\tau=5$} \\
\hline \multirow[t]{2}{*}{1} & \multirow[t]{2}{*}{$\hat{y}_{t}, \hat{c}_{t}$} & .9505 & .0128 & .0160 & .8368 & .0411 & .1040 & .0395 & .0042 & .0360 & .0100 \\
\hline & & .9511 & .0089 & .0056 & .8466 & .0504 & .0137 & .0396 & .0032 & .0083 & .0044 \\
\hline \multirow[t]{2}{*}{2} & \multirow[t]{2}{*}{$\hat{n}_{t}, \hat{y}_{t}$} & .9497 & .0138 & .0860 & .8400 & .0314 & .1880 & .0395 & .0037 & .0820 & .0060 \\
\hline & & .9508 & .0153 & .0125 & .8452 & .0470 & .0175 & .0394 & .0037 & .0123 & .0035 \\
\hline \multirow[t]{2}{*}{3} & \multirow[t]{2}{*}{$\hat{n}_{t}, \hat{c}_{t}$} & .9501 & .0107 & .0520 & .8413 & .0294 & .1580 & .0396 & .0032 & .0860 & .0000 \\
\hline & & .9502 & .0099 & .0099 & .8444 & .0409 & .0163 & .0396 & .0034 & .0125 & .0000 \\
\hline \multicolumn{12}{|c|}{ B. Simulated Method of Moments with $\tau=10$} \\
\hline \multirow[t]{2}{*}{4} & \multirow[t]{2}{*}{$\hat{y}_{t}, \hat{c}_{t}$} & .9503 & .0122 & .0300 & .8370 & .0392 & .0840 & .0393 & .0040 & .0240 & .0120 \\
\hline & & .9505 & .0095 & .0076 & .8442 & .0478 & .0124 & .0394 & .0028 & .0068 & .0049 \\
\hline \multirow[t]{2}{*}{5} & \multirow{2}{*}{$\hat{n}_{t}, \hat{y}_{t}$} & .9483 & .0133 & .0740 & .8367 & .0303 & .1320 & .0396 & .0035 & .0740 & .0020 \\
\hline & & .9497 & .0148 & .0117 & .8414 & .0417 & .0151 & .0394 & .0036 & .0117 & .0020 \\
\hline \multirow[t]{2}{*}{6} & \multirow[t]{2}{*}{$\hat{n}_{t}, \hat{c}_{t}$} & .9510 & .0101 & .0520 & .8436 & .0277 & .1640 & .0397 & .0030 & .0920 & .0020 \\
\hline & & .9516 & .0098 & .0099 & .8475 & .0377 & .0166 & .0396 & .0034 & .0129 & .0020 \\
\hline \multicolumn{12}{|c|}{ C. Simulated Methods of Moments with $\tau=20$} \\
\hline \multirow[t]{2}{*}{7} & \multirow[t]{2}{*}{$\hat{y}_{t}, \hat{c}_{t}$} & .9506 & .0120 & .0260 & .8372 & .0382 & .0880 & .0394 & .0039 & .0320 & .0120 \\
\hline & & .9509 & .0088 & .0071 & .8426 & .0443 & .0127 & .0394 & .0029 & .0079 & .0049 \\
\hline \multirow[t]{2}{*}{8} & \multirow[t]{2}{*}{$\hat{n}_{t}, \hat{y}_{t}$} & .9496 & .0131 & .1000 & .8428 & .0291 & .1420 & .0398 & .0035 & .0720 & .0020 \\
\hline & & .9510 & .0147 & .0134 & .8496 & .0426 & .0156 & .0395 & .0037 & .0116 & .0020 \\
\hline \multirow[t]{2}{*}{9} & \multirow[t]{2}{*}{$\hat{n}_{t}, \hat{c}_{t}$} & .9499 & .0101 & .0600 & .8411 & .0277 & .1260 & .0395 & .0030 & .0820 & .0000 \\
\hline & & .9502 & .0098 & .0106 & .8458 & .0376 & .0148 & .0393 & .0033 & .0123 & .0000 \\
\hline \multicolumn{12}{|c|}{ D. Generalized Method of Moments } \\
\hline \multirow[t]{2}{*}{10} & $\hat{y}_{t}, \hat{c}_{t}$ & .9501 & .0118 & .0100 & .8369 & .0375 & .1020 & .0395 & .0039 & .0180 & .0040 \\
\hline & & .9502 & .0084 & .0044 & .8429 & .0457 & .0135 & .0395 & .0026 & .0059 & .0028 \\
\hline 11 & $\hat{n}_{t}, \hat{y}_{t}$ & .9484 & .0128 & .0720 & .8382 & .0288 & .1480 & .0397 & .0034 & .0780 & .0020 \\
\hline & & .9496 & .0137 & .0116 & .8418 & .0400 & .0159 & .0396 & .0035 & .0120 & .0020 \\
\hline 12 & $\hat{n}_{t}, \hat{c}_{t}$ & .9499 & .0099 & .0540 & .8380 & .0272 & .1360 & .0395 & .0029 & .0740 & .0000 \\
\hline & & .9497 & .0094 & .0101 & .8407 & .0383 & .0153 & .0395 & .0030 & .0117 & .0000 \\
\hline
\end{tabular}

Notes: For Experiments 1, 4, 7, and 10, $\mathbf{m}_{t}=\left(\hat{y}_{t}^{2}, \hat{c}_{t}^{2}, \hat{c}_{t} \hat{y}_{t}, \hat{c}_{t} \hat{c}_{t-1}, \hat{y}_{t} \hat{y}_{t-1}\right)^{\prime}$; for Experiments 2, 5, 8, and 11, $\mathbf{m}_{t}=\left(\hat{y}_{t}^{2}, \hat{n}_{t}^{2}, \hat{n}_{t} \hat{y}_{t}, \hat{n}_{t} \hat{n}_{t-1}, \hat{y}_{t} \hat{y}_{t-1}\right)^{\prime}$; and for Experiments 3, 6, 9, and 12, $\mathbf{m}_{t}=\left(\hat{n}_{t}^{2}, \hat{c}_{t}^{2}, \hat{c}_{t} \hat{n}_{t}, \hat{c}_{t} \hat{c}_{t-1}, \hat{n}_{t} \hat{n}_{t-1}\right)^{\prime}$. See the notes to Table 1 . 


\section{Table 3. Results using Indirect Inference}

Under the Null Hypothesis

(Artificial Data is Stochastically Singular)

\begin{tabular}{|c|c|c|c|c|c|c|c|c|c|c|c|}
\hline \multicolumn{2}{|c|}{ Experiment } & $\begin{array}{c}\text { Mean } \\
\text { Median }\end{array}$ & $\begin{array}{c}\beta \\
\text { A.S.E. } \\
\text { S.D. }\end{array}$ & $\begin{array}{l}\text { Size } \\
\text { S.E. }\end{array}$ & $\begin{array}{l}\text { Mean } \\
\text { Median }\end{array}$ & $\begin{array}{c}\rho \\
\text { A.S.E. } \\
\text { S.D. }\end{array}$ & $\begin{array}{l}\text { Size } \\
\text { S.E. }\end{array}$ & $\begin{array}{l}\text { Mean } \\
\text { Median }\end{array}$ & $\begin{array}{c}\sigma \\
\text { A.S.E. } \\
\text { S.D. }\end{array}$ & $\begin{array}{l}\text { Size } \\
\text { S.E. }\end{array}$ & $\begin{array}{r}\text { OI } \\
\text { S.E. }\end{array}$ \\
\hline \multicolumn{12}{|c|}{ A. $\tau=5$} \\
\hline 1 & $\hat{y}_{t}, \hat{c}_{t}$ & .9560 & .0261 & .2700 & .8436 & .0807 & .3200 & .0400 & .0004 & .7220 & .0620 \\
\hline & & .9543 & .0295 & .0199 & .8562 & .1134 & .0209 & .0400 & .0021 & .0200 & .0108 \\
\hline \multirow[t]{2}{*}{2} & $\hat{n}_{t}, \hat{y}_{t}$ & .9499 & .0254 & .2380 & .8120 & .0195 & .2400 & .0401 & .0002 & .8480 & .2340 \\
\hline & & .9505 & .0319 & .0190 & .8519 & .1513 & 0191 & .0401 & .0021 & .0161 & .0189 \\
\hline 3 & $\hat{n}_{t}, \hat{c}_{t}$ & .9482 & .0262 & .0020 & .8390 & .0980 & .0000 & .0400 & .0006 & .6260 & .0040 \\
\hline & & .9488 & .0108 & .0020 & .8457 & .0426 & .0000 & .0399 & .0022 & .0216 & .0028 \\
\hline \multicolumn{12}{|c|}{ B. $\tau=10$} \\
\hline \multirow[t]{2}{*}{4} & $\hat{y}_{t}, \hat{c}_{t}$ & .9566 & .0252 & .2500 & .8446 & .0744 & .3160 & .0398 & .0004 & .7500 & .0540 \\
\hline & & .9574 & .0299 & .0194 & .8759 & .1172 & .0208 & .0399 & .0020 & .0194 & .0101 \\
\hline \multirow[t]{2}{*}{5} & $\hat{n}_{t}, \hat{y}_{t}$ & .9463 & .0251 & .2020 & .8020 & .0948 & 1960 & .0400 & .0002 & .8780 & .1700 \\
\hline & & .9478 & .0291 & .0180 & .8420 & .1484 & .0178 & .0399 & .0021 & .0146 & .0168 \\
\hline \multirow[t]{2}{*}{6} & $\hat{n}_{t}, \hat{c}_{t}$ & .9484 & .0255 & .0040 & .8405 & .0945 & .0000 & .0400 & .0005 & .6160 & .0020 \\
\hline & & .9489 & .0103 & .0028 & .8459 & .0405 & .0000 & .0400 & .0021 & .0218 & .0020 \\
\hline \multicolumn{12}{|c|}{ C. $\tau=20$} \\
\hline \multirow[t]{2}{*}{7} & $\hat{y}_{t}, \hat{c}_{t}$ & .9559 & .0246 & .3060 & .8366 & .0701 & .3660 & .0400 & .0003 & .7840 & .0600 \\
\hline & & .9564 & .0327 & .0206 & .8724 & .1283 & 0215 & .0400 & .0020 & .0184 & .0106 \\
\hline \multirow[t]{2}{*}{8} & $\hat{n}_{t}, \hat{y}_{t}$ & .9448 & .0254 & .2040 & .7854 & .0970 & 2000 & .0398 & .0002 & .8680 & .2280 \\
\hline & & .9489 & .0316 & .0180 & .8460 & .1838 & 0179 & .0397 & .0019 & .0151 & .0188 \\
\hline \multirow[t]{2}{*}{9} & $\hat{n}_{t}, \hat{c}_{t}$ & .9488 & .0251 & .0100 & .8414 & .0925 & .0020 & .0401 & .0005 & .5840 & .0040 \\
\hline & & .9487 & .0109 & .0044 & .8451 & .0433 & .0020 & .0401 & .0020 & .0220 & .0028 \\
\hline
\end{tabular}

Notes: For Experiments 1, 4, and 7, the VAR consists of $\hat{y}_{t}$ and $\hat{c}_{t}$; for Experiments 2, 5, and 8, the VAR consists of $\hat{y}_{t}$ and $\hat{n}_{t}$; and for Experiments 3,6, and 9, the VAR consists of $\hat{n}_{t}$, and $\hat{c}_{t}$. In all cases a VAR of order one is used. See the notes to Table 1. 
Table 4. Maximum Likelihood Results

Artificial Data is Nonsingular Because True Model has

Multiple Structural Shocks

\begin{tabular}{|c|c|c|c|c|c|c|c|c|c|c|}
\hline \multirow{2}{*}{\multicolumn{2}{|c|}{ Experiment }} & \multicolumn{3}{|c|}{$\bar{\beta}$} & \multicolumn{3}{|c|}{$\rho$} & \multicolumn{3}{|c|}{$\sigma$} \\
\hline & & $\begin{array}{c}\text { Mean } \\
\text { Bias }\end{array}$ & $\begin{array}{c}\text { A.S.E. } \\
\text { S.D. }\end{array}$ & $\begin{array}{l}\text { Size } \\
\text { S.E. }\end{array}$ & $\begin{array}{c}\text { Mean } \\
\text { Bias }\end{array}$ & $\begin{array}{c}\text { A.S.E. } \\
\text { S.D. }\end{array}$ & $\begin{array}{l}\text { Size } \\
\text { S.E. }\end{array}$ & $\begin{array}{c}\text { Mean } \\
\text { Bias }\end{array}$ & $\begin{array}{c}\text { A.S.E. } \\
\text { S.D. }\end{array}$ & $\begin{array}{l}\text { Size } \\
\text { S.E. }\end{array}$ \\
\hline \multicolumn{11}{|c|}{ A. Using as Many Variables as Shocks } \\
\hline \multirow[t]{2}{*}{1} & $\hat{y}_{t}$ & .8392 & .0418 & 1260 & -.0195 & .1157 & .9920 & .2353 & .0554 & .9900 \\
\hline & & -.1108 & .2323 & .0148 & -.8695 & .2468 & .0040 & .1923 & .0812 & .0044 \\
\hline \multirow[t]{2}{*}{2} & $\hat{c}_{t}$ & .9098 & .0942 & 1240 & .8132 & .1209 & 1040 & .0547 & .0383 & .1300 \\
\hline & & -0.0402 & .1189 & .0147 & -.0368 & .1273 & .0137 & .0147 & .0372 & .0150 \\
\hline \multirow[t]{2}{*}{3} & $\hat{n}_{t}$ & .6067 & 1835 & .5200 & .0381 & 1479 & .9840 & .1199 & .0222 & .9840 \\
\hline & & -.3433 & .2334 & .0223 & -.8019 & .1878 & .0056 & .0799 & .0236 & .0056 \\
\hline \multicolumn{11}{|c|}{ B. Incorporating Bayesian Priors } \\
\hline \multirow[t]{2}{*}{4} & $\hat{y}_{t}$ & .9303 & .0268 & .0020 & .7657 & .0876 & .0820 & .1890 & .0285 & .9860 \\
\hline & & -.0197 & .0162 & .0020 & -.0843 & .0589 & .0123 & 1490 & .0156 & .0053 \\
\hline \multirow[t]{2}{*}{5} & $\hat{c}_{t}$ & .9482 & .0227 & .0020 & .8429 & .0092 & .0020 & .0424 & .0092 & .0000 \\
\hline & & -.0018 & .0069 & .0020 & -.0071 & .0027 & .0020 & .0024 & .0027 & .0000 \\
\hline \multirow[t]{2}{*}{6} & $\hat{n}_{t}$ & .9232 & .0319 & .0020 & .7331 & .0968 & 1340 & .2011 & .0313 & .9780 \\
\hline & & -0.0268 & .0138 & .0020 & -0.1169 & .0523 & .0152 & .1611 & .0149 & .0066 \\
\hline \multicolumn{11}{|c|}{ C. Adding Measurement Errors } \\
\hline \multirow[t]{2}{*}{7} & $\hat{y}_{t}, \hat{c}_{t}$ & .9999 & .0053 & .9780 & .9765 & .0095 & .9900 & .0544 & .0048 & .8960 \\
\hline & & .0499 & .0006 & .0066 & .0265 & .0037 & .0044 & .0144 & .0034 & .0137 \\
\hline \multirow[t]{2}{*}{8} & $\hat{n}_{t}, \hat{y}_{t}$ & .0394 & .0930 & .8920 & .7820 & .0928 & .0620 & .0298 & .0081 & 1.000 \\
\hline & & -.9106 & 1344 & .0139 & -.0680 & .0672 & .0108 & -.0102 & .0064 & .0000 \\
\hline \multirow[t]{2}{*}{9} & $\hat{n}_{t}, \hat{c}_{t}$ & .9554 & .0020 & .6760 & .7748 & .0245 & .7940 & .0508 & .0028 & 1.000 \\
\hline & & .0054 & .0051 & .0209 & -.0752 & .0363 & .0181 & .0108 & .0029 & .0000 \\
\hline
\end{tabular}

Notes: Bias is the arithmetic average of the difference between the estimated and true parameter values. See the notes to Table 1 . 
Table 5. Results using Methods of Moments

Artificial Data is Nonsingular Because True Model has

Multiple Structural Shocks

\begin{tabular}{|c|c|c|c|c|c|c|c|c|c|c|}
\hline \multirow{2}{*}{\multicolumn{2}{|c|}{ Experiment }} & \multicolumn{3}{|c|}{$\beta$} & \multicolumn{3}{|c|}{$\rho$} & \multicolumn{3}{|c|}{$\sigma$} \\
\hline & & Mean & A.S.E. & Size & Mean & A.S.E. & Size & Mean & A.S.E. & Size \\
\hline$\#$ & Var. & Bias & S.D. & S.E. & Bias & S.D. & S.E. & Bias & S.D. & S.E. \\
\hline \multicolumn{11}{|c|}{ A. Simulated Method of Moments with $\tau=5$} \\
\hline \multirow[t]{2}{*}{1} & $\hat{y}_{t}, \hat{c}_{t}$ & .9484 & .0117 & .0200 & .8122 & .0430 & .1500 & .0449 & .0042 & .1300 \\
\hline & & -.0016 & .0088 & .0063 & -.0378 & .0530 & .0160 & .0049 & .0033 & .0150 \\
\hline \multirow[t]{2}{*}{2} & $\hat{n}_{t}, \hat{y}_{t}$ & .9705 & .0096 & .5900 & .7846 & .0366 & .3940 & .0453 & .0036 & .3080 \\
\hline & & .0205 & .0126 & .0220 & -.0654 & .0508 & .0219 & .0053 & .0040 & .0206 \\
\hline \multirow[t]{2}{*}{3} & $\hat{n}_{t}, \hat{c}_{t}$ & .9512 & .0106 & .1080 & .7552 & .0380 & .6200 & .0530 & .0038 & .9040 \\
\hline & & .0012 & .0121 & .0139 & -.0948 & .0544 & .0217 & .0130 & .0046 & .0132 \\
\hline \multicolumn{11}{|c|}{ B. Simulated Method of Moments with $\tau=10$} \\
\hline \multirow[t]{2}{*}{4} & $\hat{y}_{t}, \hat{c}_{t}$ & .9479 & .0112 & .0036 & .8081 & .0418 & .1860 & .0446 & .0040 & .1240 \\
\hline & & -.0021 & .0093 & .0083 & -.0419 & .0547 & .0174 & .0046 & .0033 & .0147 \\
\hline \multirow[t]{2}{*}{5} & $\hat{n}_{t}, \hat{y}_{t}$ & .9705 & .0091 & .6060 & .7869 & .0351 & .4160 & .0454 & .0034 & .3640 \\
\hline & & .0205 & .0116 & .0219 & -.0631 & .0471 & .0220 & .0054 & .0041 & .0215 \\
\hline \multirow[t]{2}{*}{6} & $\hat{n}_{t}, \hat{c}_{t}$ & .9518 & .0102 & .1140 & .7545 & .0363 & .7180 & .0528 & .0037 & .9280 \\
\hline & & .0018 & .0116 & .0142 & -0.955 & .0515 & .0201 & .0128 & .0043 & .0116 \\
\hline \multicolumn{11}{|c|}{ C. Simulated Methods of Moments with $\tau=20$} \\
\hline \multirow[t]{2}{*}{7} & $\hat{y}_{t}, \hat{c}_{t}$ & .9477 & .0110 & .0160 & .8095 & .0407 & .1640 & .0448 & .0040 & .1220 \\
\hline & & -.0023 & .0082 & .0056 & -.0405 & .0523 & .0166 & .0048 & .0030 & .0146 \\
\hline \multirow[t]{2}{*}{8} & $\hat{n}_{t}, \hat{y}_{t}$ & .9699 & .0090 & .6040 & .7871 & .0338 & .4100 & .0458 & .0033 & .4140 \\
\hline & & .0199 & .0117 & .0219 & -.0629 & .0480 & .0220 & .0058 & .0041 & .0220 \\
\hline \multirow[t]{2}{*}{9} & $\hat{n}_{t}, \hat{c}_{t}$ & .9512 & .0100 & .0880 & .7566 & .0358 & .7040 & .0532 & .0036 & .9520 \\
\hline & & .0012 & .0109 & .0127 & -.0934 & .0464 & .0204 & .0132 & .0041 & .0096 \\
\hline \multicolumn{11}{|c|}{ D. Generalized Method of Moments } \\
\hline \multirow[t]{2}{*}{10} & $\hat{y}_{t}, \hat{c}_{t}$ & .9476 & .0108 & .0320 & .8104 & .0397 & .1520 & .0449 & .0039 & .1380 \\
\hline & & -.0024 & .0083 & .0079 & -.0396 & .0501 & .0161 & .0049 & .0030 & .0154 \\
\hline \multirow[t]{2}{*}{11} & $\hat{n}_{t}, \hat{y}_{t}$ & .9704 & .0087 & .6040 & .7875 & .0330 & .4140 & .0457 & .0033 & .4300 \\
\hline & & .0204 & .0114 & .0219 & -.0625 & .0473 & .0220 & .0057 & .0473 & .0221 \\
\hline \multirow[t]{2}{*}{12} & $\hat{n}_{t}, \hat{c}_{t}$ & .9508 & .0098 & .1080 & .7569 & .0342 & .9400 & .0530 & .0035 & .9400 \\
\hline & & .0008 & .0112 & .0139 & -.0931 & .0507 & .0106 & .0130 & .0042 & .0106 \\
\hline
\end{tabular}

Notes: See the notes to Tables 1 and 2. 
Table 6. Results using Indirect Inference

Artificial Data is Nonsingular Because True Model has

Multiple Structural Shocks

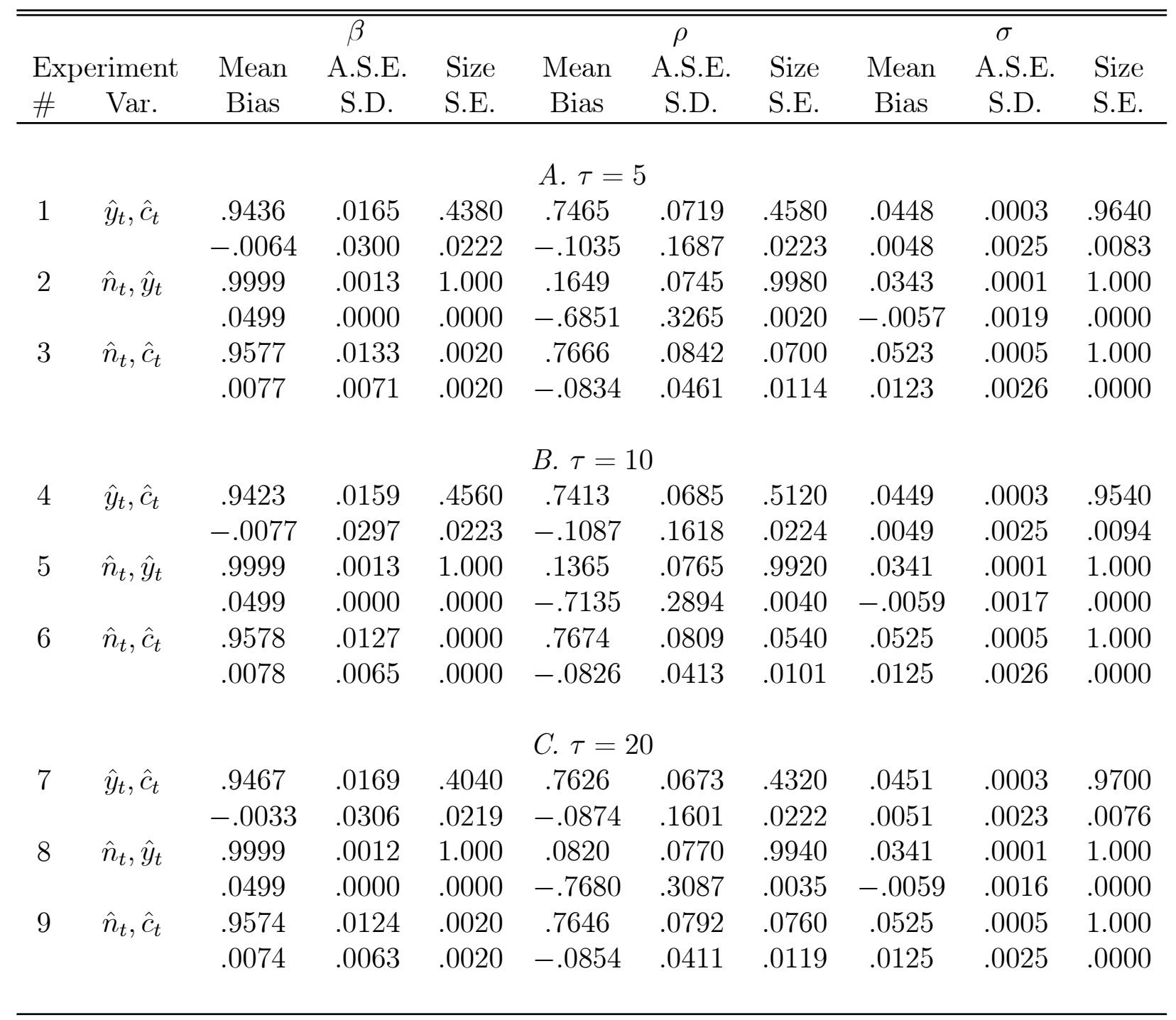

Notes: See the notes to Tables 1 and 3. 


\section{Table 7. Comparison in Terms of Computing Time}

\begin{tabular}{lccc}
\hline \hline \multicolumn{1}{c}{ Method } & & $\begin{array}{c}\text { Seconds } \\
\text { per Replication }\end{array}$ & $\begin{array}{c}\text { Compared with } \\
\text { GMM }\end{array}$ \\
\hline \multirow{3}{*}{ Maximum Likelihood } & - & 3.19 & 4.8 \\
Simulated Method of Moments & 5 & 7.52 & 11.2 \\
& 10 & 14.74 & 22.0 \\
Indirect Inference & 20 & 27.41 & 40.1 \\
& 5 & 27.89 & 41.6 \\
Generalized Method of Moments & - & 59.15 & 88.3 \\
& 20 & 90.96 & 135.8 \\
& & & \\
\hline
\end{tabular}

Notes: The Monte Carlo was performed using GAUSS for Windows running in a Dell Inspiron 7500 with Pentium III processor. 


\section{A Appendix A : The Log-linearized Model}

In what follows, variables without time subscript denote steady state values and the circumflex denotes percentage deviation from steady state. For example, $\hat{c}_{t}=\left(c_{t}-c\right) / c$ is the percentage deviation of consumption from its steady state at time $t$. For the model in Section 2, the linearized first-order conditions of the agent's problem are (notice that the marginal products of labor and capital have already been substituted out):

$$
\begin{aligned}
E_{t} \hat{c}_{t+1} & =\hat{c}_{t}+\varsigma(\alpha-1) E_{t} \hat{k}_{t+1}+\varsigma(1-\alpha) E_{t} \hat{n}_{t+1}+\varsigma E_{t} \hat{z}_{t+1} \\
\hat{n}_{t} & =-(1 / \alpha) \hat{c}_{t}+\hat{k}_{t}+(1 / \alpha) \hat{z}_{t} .
\end{aligned}
$$

where $\varsigma=\alpha \beta(k / n)^{\alpha-1}$ and the steady-state capital-labor ratio $k / n=((1 / \beta+\delta-1) / \alpha)^{1 /(\alpha-1)}$. The linearized production function and resource constraint are:

$$
\begin{aligned}
& \hat{y}_{t}=\alpha \hat{k}_{t}+(1-\alpha) \hat{n}_{t}+\hat{z}_{t}, \\
& \hat{y}_{t}=\gamma \hat{c}_{t}+(1-\gamma) \hat{x}_{t}
\end{aligned}
$$

where $\gamma$ is the consumption-output ratio in steady and equals $1-\delta(k / n)^{1-\alpha}$. Finally, the linearized law of motions for capital and the technology shock are:

$$
\begin{aligned}
\hat{k}_{t+1} & =(1-\delta) \hat{k}_{t}+\delta \hat{x}_{t}, \\
\hat{z}_{t+1} & =\rho \hat{z}_{t}+\epsilon_{t} .
\end{aligned}
$$




\section{References}

[1] Altug, S., (1989), "Time-to-Build and Aggregate Fluctuations: Some New Evidence," International Economic Review 30, 889-920.

[2] Ambler, S., Guay, A., and Phaneuf, L., (1999), "Wage Contracts and Labor Adjustment Costs as Endogenous Propagation Mechanisms," Université du Québec à Montréal, Working Paper 69.

[3] Bencivenga, V. R., (1992), "An Econometric Study of Hours and Output Variation with Preference Shocks," International Economic Review 33, 449-471.

[4] Bergin, P. R., (2003), "Putting the 'New Open Economy Macroeconomics' to a Test," Journal of International Economics 60, 3-34.

[5] Blanchard, O. J. and Kahn, C. M., (1980), "The Solution of Linear Difference Models under Rational Expectations," Econometrica, 48, 1305-1311.

[6] Bouakez, H., Cardia, E., and Ruge-Murcia, F. J., (2001), "Habit Formation and the Persistence of Monetary Shocks," Université de Montréal, Mimeo.

[7] Braun, R. A., (1994), "Tax Disturbances and Real Economic Activity in the Postwar United States?" Journal of Monetary Economics 33, 441-462.

[8] Browning, M., Hansen, L. P., and Heckman, J. J., (1999), "Micro Data and General Equilibrium Models," in Handbook of Macroeconomics edited by J. Taylor and M. Woodford. North-Holland: Amsterdam.

[9] Burnside, C., Eichenbaum, M., and Rebelo, S., (1993), "Labor Hoarding and the Business Cycle," Journal of Political Economy 101, 245-273.

[10] Chang, Y., Gomes, J. F., and Schofheide, F., (2002), "Learning-by-Doing as a Propagation Mechanism," American Economic Review 92, 1498-1520.

[11] Christiano, L. J., (1988), "Why Does Inventory Investment Fluctuate So Much?" Journal of Monetary Economics 21, 247-280.

[12] Christiano, L. J. and Eichenbaum, M., (1992), "Current Real-Business Cycle Theories and Aggregate Labor-Market Fluctuations," American Economic Review, 82, 430-450.

[13] Christiano, L. J. and den Haan, W. J., (1996), "Small-Sample Properties of GMM for Business-Cycle Analysis" Journal of Business and Economic Statistics 14, 309327. 
[14] Christiano, L. J., Eichenbaum, M., and Evans, C. L., (2001), "Nominal Rigidities and the Dynamic Effects of a Shock to Monetary Policy," NBER Working Paper 8403.

[15] DeJong, D. N., Ingram, B. F., and Whiteman, C. H., (1996), "A Bayesian Approach to Calibration," Journal of Business and Economic Statistics 14, 1-9.

[16] DeJong, D. N., Ingram, B. F., and Whiteman, C. H., (2000), "A Bayesian Approach to Dynamic Macroeconomics," Journal of Econometrics 98, 203-223.

[17] Dib, A. and Phaneuf, L., (2001), "An Econometric U.S. Business Cycle Model with Nominal and Real Rigidities," Université du Québec à Montréal, Working Paper 137.

[18] Duffie, D. and Singleton, K. J., (1993), "Simulated Moments Estimation of Markov Models of Asset Prices," Econometrica 61, 929-952.

[19] Fernández-Villaverde, J. and Rubio, J., (2002), "Estimating Nonlinear Dynamic Equilibrium Economies: A Likelihood Approach," University of Pennsylvania, Mimeo.

[20] Fuhrer, J. C., Moore, G. R., and Schuh, S. D., (1995), "Estimating the Linear-Quadratic Inventory Model: Maximum Likelihood versus Generalized Method of Moments," Journal of Monetary Economics 35, 115-157.

[21] Gouriéroux, C., Monfort, A., and Renault, E., (1993), "Indirect Inference," Journal of Applied Econometrics 8, S85-S118.

[22] Gregory, A. and Smith, G., (1991), "Calibration as Testing: Inference in Simulated Macroeconomic Models," Journal of Business and Economic Statistics 9, 297-304.

[23] Hall, G. J., (1996), "Overtime, Effort, and the Propagation of Business Cycle Shocks," Journal of Monetary Economics 38, 139-160.

[24] Hamilton, J. D., (1994), Time Series Analysis, Princeton: Princeton University Press.

[25] Hansen, G. D., (1985), "Indivisible Labor and the Business Cycle," Journal of Monetary Economics, 16, 309-327.

[26] Hansen, L. P., (1982), "Large Sample Properties of Generalized Method of Moments Estimators," Econometrica 50, 1929-1954.

[27] Hansen, L. P. and Sargent, T. J., (1980), "Formulating and Estimating Dynamic Linear Rational Expectations Models," Journal of Economic Dynamics and Control 2, 7-46. 
[28] Hansen, L. P. and Heckman, J. J., (1996), "The Empirical Foundations of Calibration," Journal of Economic Perspectives 10, 87-104.

[29] Hansen, L. P. and Sargent, T. J., (1998), "Recursive Linear Models of Dynamic Economies," University of Chicago, Mimeo.

[30] Ingram, B. F., Kocherlakota, N., and Savin, N. E., (1994), "Explaining Business Cycles: A Multiple-Shock Approach," Journal of Monetary Economics 34, 415-428.

[31] Ireland, P., (1999), "A Method for Taking Models to the Data," Boston College, Mimeo.

[32] Ireland, P., (2001), "Sticky Price Models of the Business Cycle: Specification and Stability," Journal of Monetary Economics 47, 3-18.

[33] Judge, G. G., Griffiths, W. E., Carter Hill, R., Lütkepohl, H., and Lee, T.-C., (1985), The Theory and Practice of Econometrics, John Wiley and Sons: New York.

[34] Kalman, R. E., (1980), "A New Approach to Linear Filtering and Prediction Problems," Journal of Basic Engineering, Transactions of the ASME Series D 82, 35-45.

[35] Kydland, F. E., and Prescott, E. C., (1996), "The Computational Experiment: An Econometric Tool," Journal of Economic Perspectives 10, 69-85.

[36] Kim, J., (2000), "Constructing and Estimating a Realistic Optimizing Model of Monetary Policy," Journal of Monetary Economics 45, 329-359.

[37] King, R. G., Plosser, C. I., and Rebelo, S., (1988), "Production, Growth, and Bussines Cycles: The Basic Neoclassical Model," Journal of Monetary Economics, 21, 195-232.

[38] Lee, B.-S., and Ingram, B. F., (1991), "Simulation Estimation of Time-Series Models," Journal of Econometrics 47, 195-205.

[39] Leeper, E. M. and Sims, C. A., (1994), "Toward a Modern Macroeconomic Model Usable for Policy Analysis" NBER Macroeconomics Annual 1994, 81-118.

[40] McFadden, D., (1986), "A Method of Simulated Moments for Estimation of Discrete Response Models without Numerical Integration," Econometrica, 57, 995-1026.

[41] McGrattan, E. R., (1994), "The Macroeconomic Effects of Distortionary Taxation," Journal of Monetary Economics 33, 573-601. 
[42] McGrattan, E. R., Rogerson, R., and Wright, R., (1997), "An Equilibrium Model of the Business Cycle with Household Production and Fiscal Policy," International Economic Review 38, 267-290.

[43] Newey, W. K., (1985), "Generalized Method of Moments Specification Testing," Journal of Econometrics 29, 229-256.

[44] Pakes, A. and Pollard, D., (1989), "The Asymptotic Distribution of Simulation Experiments," Econometrica, 57, 1027-1057.

[45] Ripley, B. D., (1987), Stochastic Simulation, John Wiley \& Sons: New York.

[46] Ruge-Murcia, F. J., (2002), "Methods to Estimate Dynamic Stochastic General Equilibrium Models," University of California at San Diego, Economics Discussion Paper 2002-18.

[47] Sargent, T., (1989), "Two Models of Measurement and the Investment Accelerator," Journal of Political Economy 97, 251-287.

[48] Sims, C., (1997), "Solving Linear Rational Expectations Models," Yale University, Mimeo.

[49] Smith, A. A., (1993), "Estimating Nonlinear Time-Series Models using Simulated Vector Autoregressions," Journal of Applied Econometrics 8, 63-84.

[50] Stone, J. R. N., (1954), The Measurement of Consumers' Expenditure and Behaviour in the United Kingdom, Cambridge University Press: Cambridge.

[51] Theil, H. and Goldberger, A. S., (1961), International Economic Review 2, 65-78.

[52] Watson, M., (1993), "Measures of Fit for Calibrated Models," Journal of Political Economy 101, 1011-1041. 

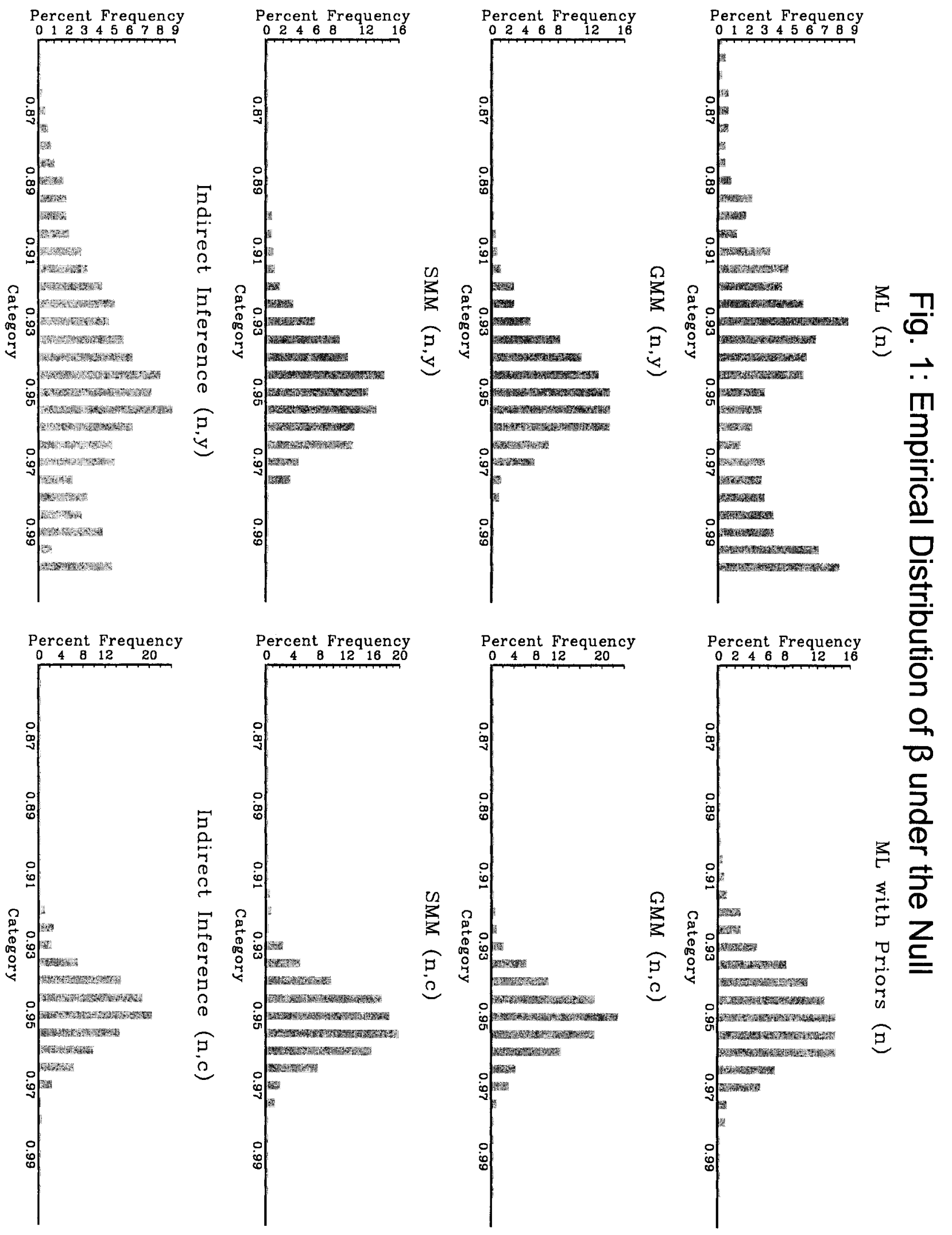

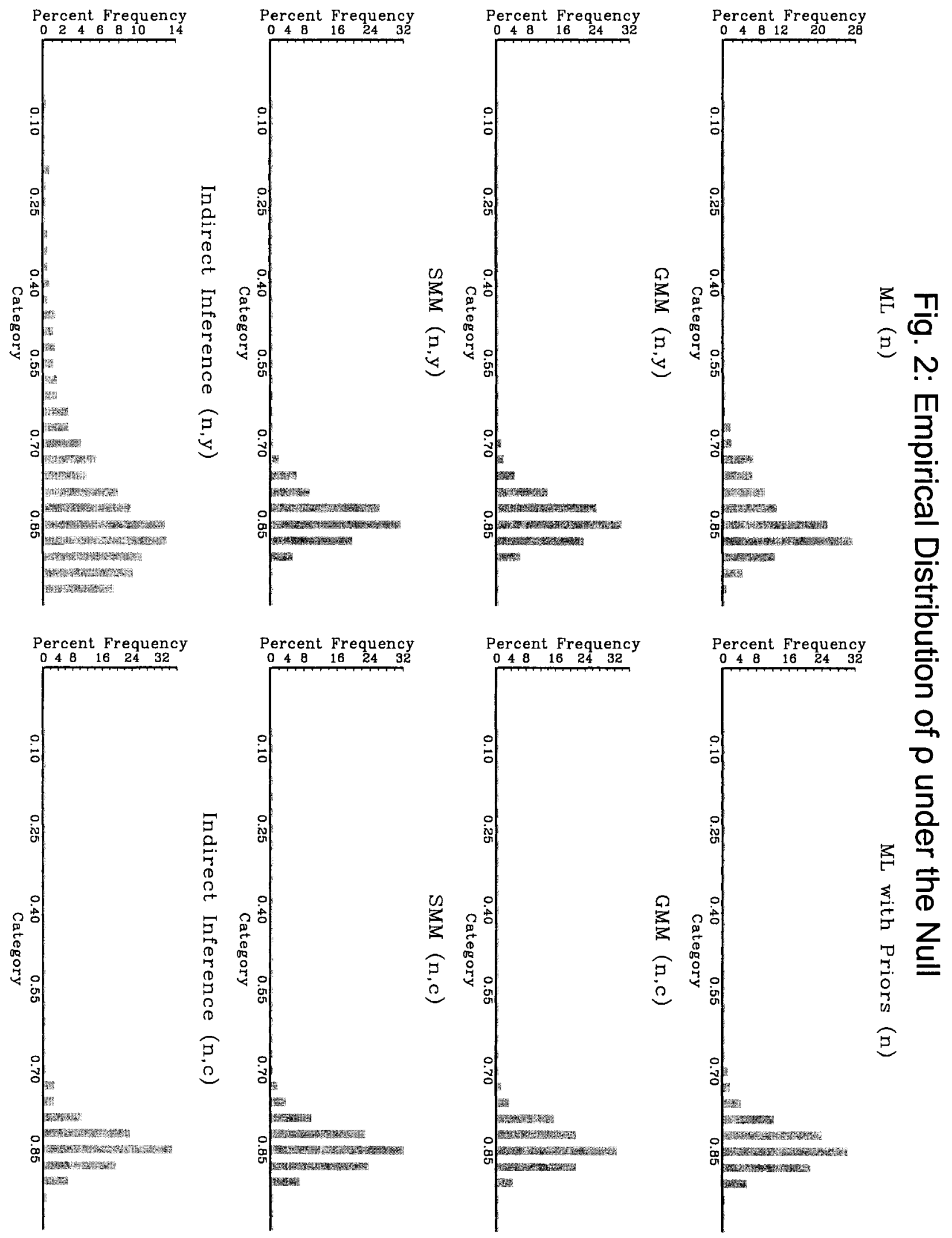

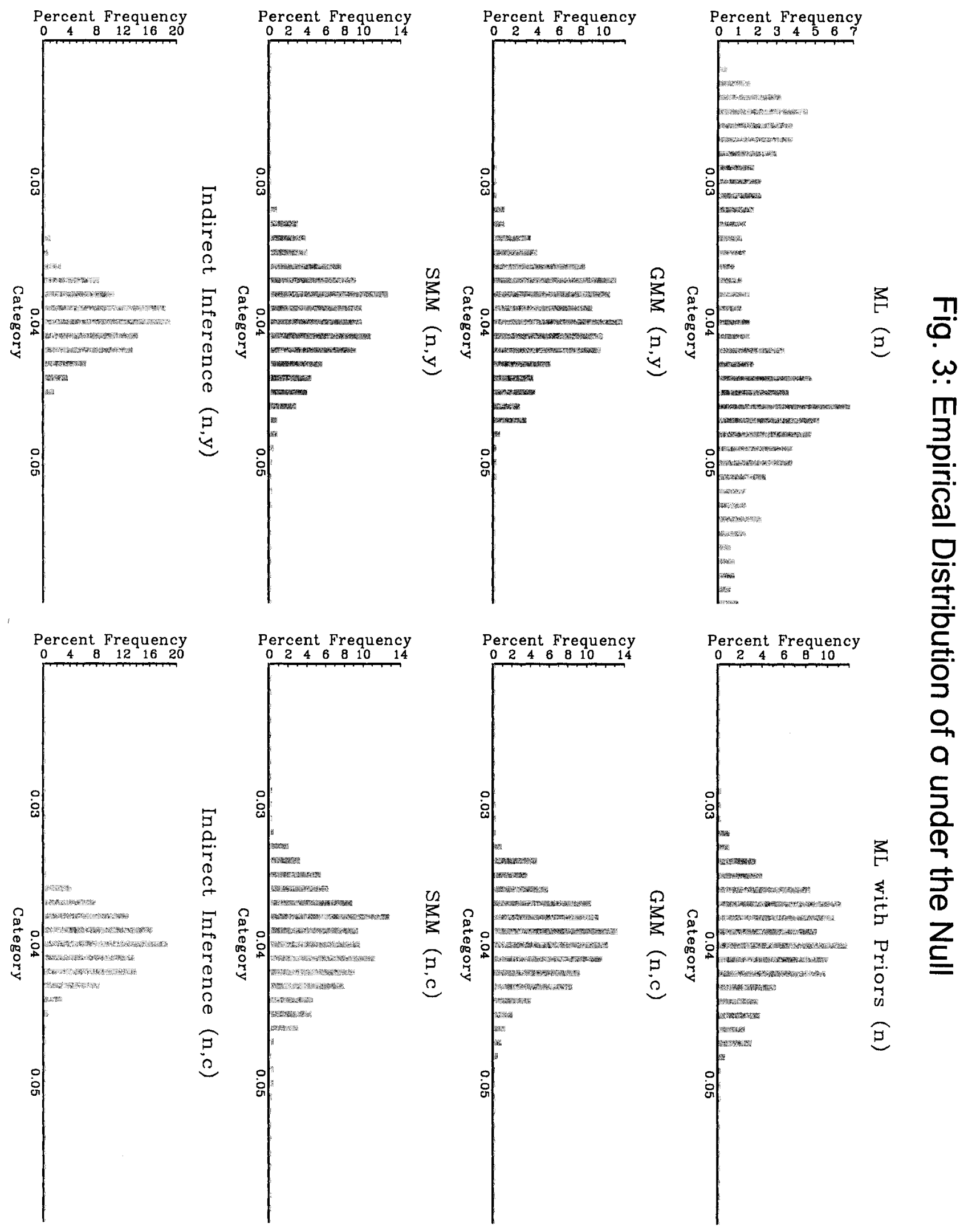

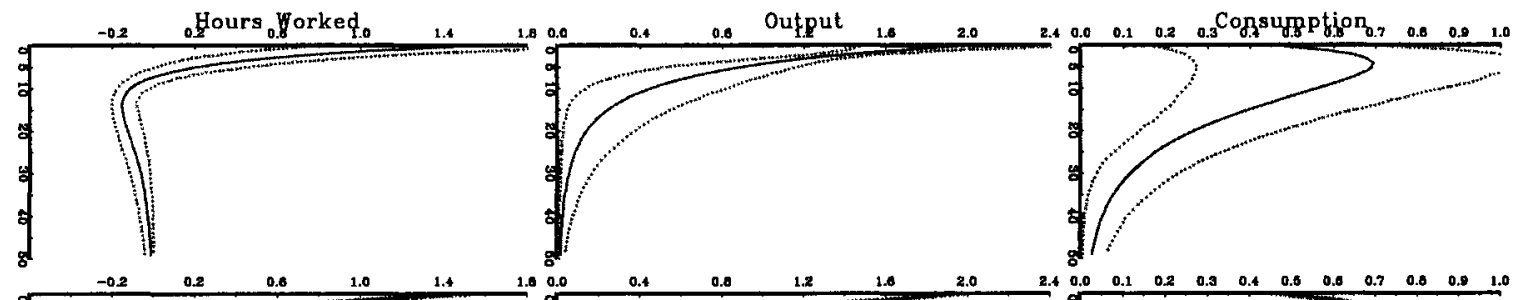

可
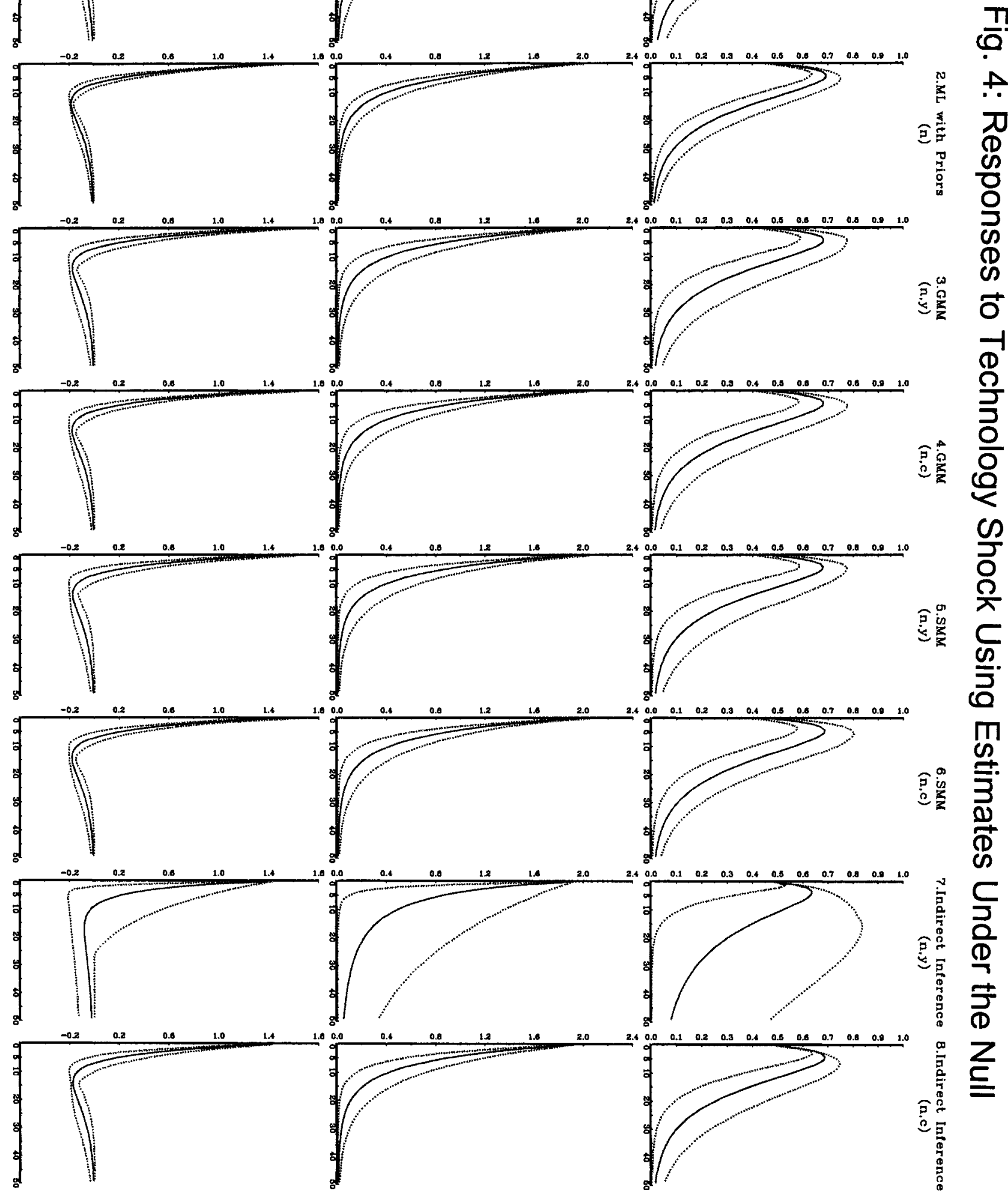

\&

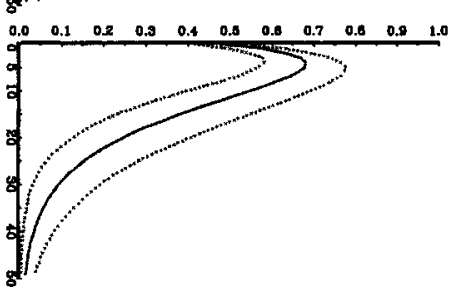

$\leqslant \frac{2}{2}$
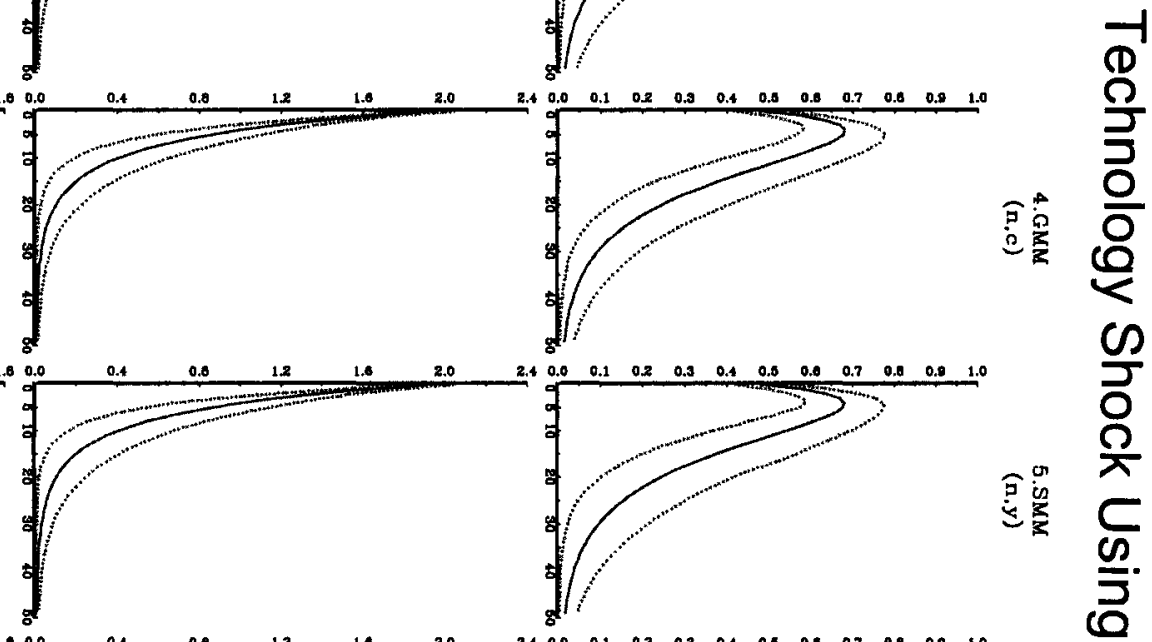


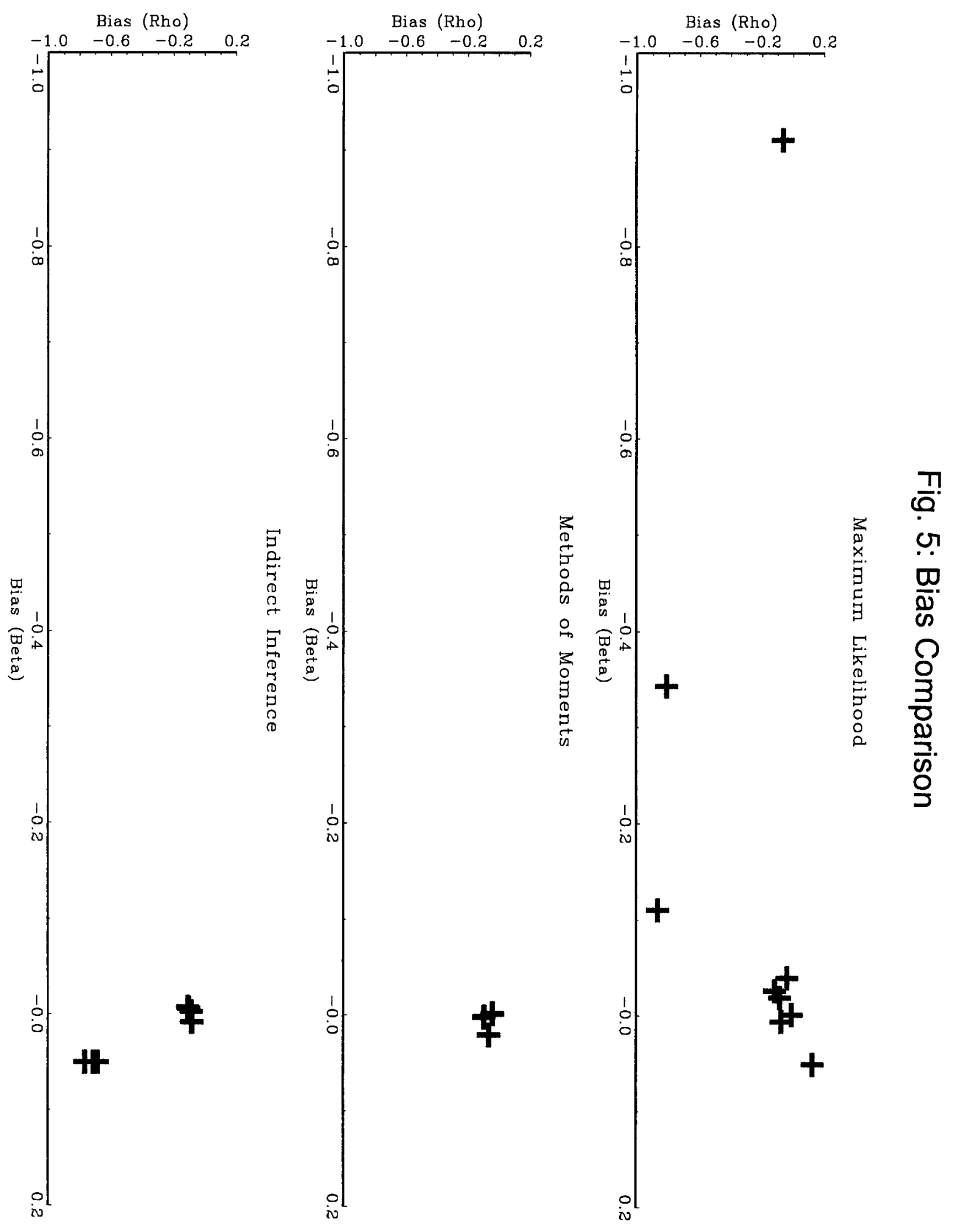




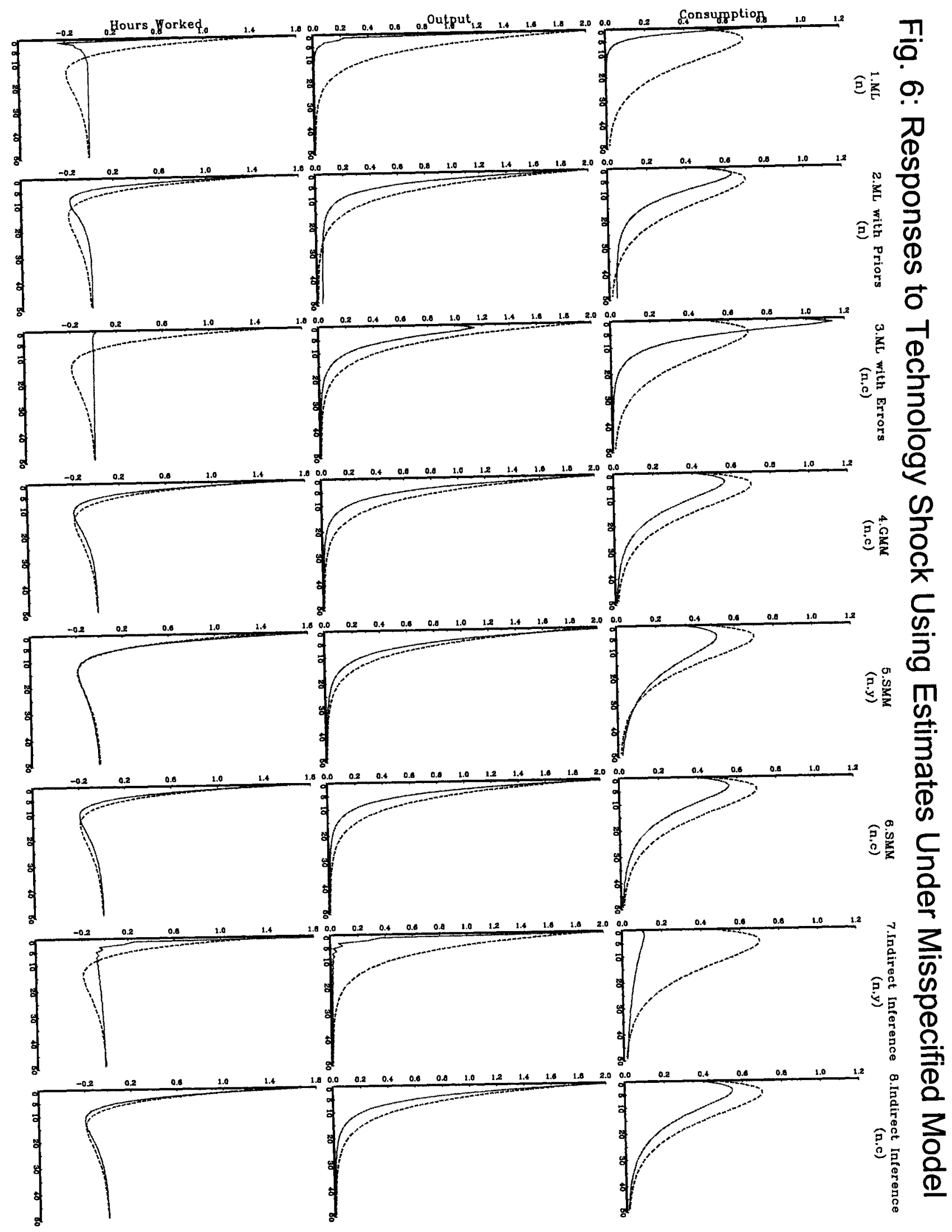

